\title{
Catalytic Asymmetric Synthesis of Allylic Thiol Derivatives
}

\author{
Larry E. Overman, ${ }^{*}$ Scott W. Roberts, and Helen F. Sneddon \\ Department of Chemistry, 1102 Natural Sciences II, University of California, \\ Irvine, California 92697-2025, USA
}

\section{Supporting Information - Table of Contents}

Materials and Methods

Experimental Procedures

NMR Spectra

SFC and GC Traces used to Determine Enantiomeric Purity

\begin{abstract}
Materials and Methods.
$[(R p, S)-\mathrm{COP}-\mathrm{Cl}]_{2}$ (di- $\mu$-chlorobis $\left[\left(\eta^{5}-(R p, S)-2-\left(2^{\prime}-\left(4^{\prime}-\right.\right.\right.\right.$ methylethyl)oxazolinyl)cyclopentadienyl, 1-C,3'-N)( $\eta^{4}$-tetraphenylcyclo-butadiene)cobalt]dipalladium, 1) and its enantiomer, $[(\mathrm{Sp}, \mathrm{R})-\mathrm{COP}-\mathrm{Cl}]_{2}$, were prepared according to published procedures. ${ }^{1}$ Both enantiomers are commercially available from Aldrich Chemical Co. (product \# 661791 and 646636). COP-complexes $\mathbf{3}^{2}{ }^{2} \mathbf{4}^{1}$ and $\mathbf{5}^{2}$ were prepared from $[(R p, S) \text {-COP-OAc }]_{2}{ }^{1}$ Rearrangements with COP complexes were performed in anhydrous solvents (either freshly distilled or passed through activated-alumina columns) in oven-dried 2-dram vials, which were sealed with Thermoset Teflon ${ }^{(B}$ PTFE-lined caps. Heating was performed in a multi-bored aluminum block placed on a IKAmag temperature modulator with the probe immersed in a vial well of the aluminum block filled with mineral oil.

Thin layer chromatography (TLC) was conducted with E. Merck silica gel 60 F254 pre-coated plates, $(0.25 \mathrm{~mm})$ and visualized using a combination of $\mathrm{UV}$, anisaldehyde, ceric ammonium molybdate, iodine on silica, and potassium permanganate staining. E. Merck silica gel (particle size 0.040-0.063 mm) was used for flash chromatography. HPLC determination of enantiopurity was carried out on a Mettler Toledo SFC (super critical $\mathrm{CO}_{2}$ liquid chromatography) using either Chiral OB-H, or AD
\end{abstract}

\footnotetext{
${ }^{1}$ (a) Anderson, C. E.; Kirsch, S. F.; Overman, L. E.; Richards, C. J.; Watson, M. P. Org. Synth. 2007, 84, 148-155. (b) Anderson, C. E.; Overman, L. E.; Richards, C. J.; Watson, M. P; White, N. Org. Synth. 2007, $84,139-147$.

2 The preparation and characterization of this COP complex will be described in a forthcoming publication.
} 
columns. GC determination of enantiopurity was carried out on a Agilent 6850 series II network GC system using a Cyclosil-B capillary $30.0 \mathrm{~m}$ x $250 \mu \mathrm{m}$ x $0.25 \mu \mathrm{m}$ nominal column. ${ }^{1} \mathrm{H}$ NMR spectra were recorded at 500 or $600 \mathrm{MHz}$ and are reported relative to signals of the deuterated solvent. IR spectra were recorded as thin films using an Applied Systems REACT-IR 1000 spectrometer. Optical rotations were measured with a Jasco P1010 polarimeter. High resolution mass spectra were obtained at the UC Irvine Mass Spectrometry Facility using a Fisions Autospec spectrometer. Concentrations were carried out at reduced pressure using a rotary evaporator.

\section{Experimental Procedures.}

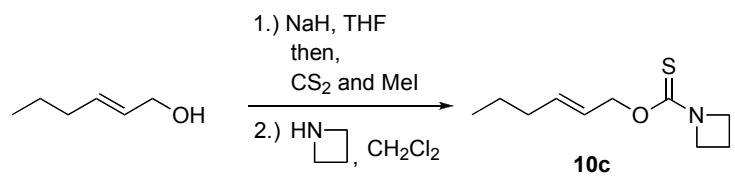

(E)-O-2-Hexenyl 1-azetinylcarbamothioate (10c). Sodium hydride (1.20 g, 30.0 mmol, 3 equiv) was added to a solution of trans-hex-2-en-1-ol (1.00 g, $10.0 \mathrm{mmol})$ and THF $(20 \mathrm{~mL})$ at room temperature. After $1.5 \mathrm{~h}, \mathrm{CS}_{2}(0.6 \mathrm{~mL}, 10 \mathrm{mmol}, 1$ equiv) was added, followed by the dropwise addition of $\mathrm{MeI}(0.83 \mathrm{~mL}, 13 \mathrm{mmol}, 1.3$ equiv). After 3 $\mathrm{h}$, saturated aqueous $\mathrm{NH}_{4} \mathrm{Cl}(20 \mathrm{~mL})$ was added to the yellow solution, and the aqueous layer was extracted with EtOAc $(3 \times 20 \mathrm{~mL})$. The combined organic extracts were washed with $\mathrm{H}_{2} \mathrm{O}$, brine, dried $\left(\mathrm{MgSO}_{4}\right)$, and concentrated to provide $1.89 \mathrm{~g}$ of the crude xanthate as a yellow oil, which was used without further purification.

Azetidine $(0.1 \mathrm{~mL}, 1.5 \mathrm{mmol})$ was added to a solution of a portion $(280 \mathrm{mg}, 1.47$ mmol) of this crude methylxanthate and hexanes $(0.5 \mathrm{~mL})$, and the resulting solution was maintained at room temperature for $30 \mathrm{~min}$. Concentration and purification of the residue by silica gel chromatography $\left(100 \%\right.$ hexanes, $2 \% \mathrm{Et}_{2} \mathrm{O}$-hexanes, $5 \% \mathrm{Et}_{2} \mathrm{O}$-hexanes, $10 \%$ $\mathrm{Et}_{2} \mathrm{O}$-hexanes) provided $O$-carbamothioate $10 \mathrm{c}(285 \mathrm{mg}, 97 \%)$ as a colorless oil: $R_{f} 0.26$ (10\% $\mathrm{Et}_{2} \mathrm{O}$-hexanes); ${ }^{1} \mathrm{H} \mathrm{NMR}\left(500 \mathrm{MHz}, \mathrm{CDCl}_{3}\right) \delta 5.78(\mathrm{dt}, J=15.3,6.7 \mathrm{~Hz}, 1 \mathrm{H}), 5.62$ $(\mathrm{dt}, J=15.3,6.4 \mathrm{~Hz}, 1 \mathrm{H}) ; 4.86(\mathrm{~d}, J=6.4 \mathrm{~Hz}, 2 \mathrm{H}), 4.19(\mathrm{t}, J=7.6 \mathrm{~Hz}, 2 \mathrm{H}), 4.10(\mathrm{t}, J=$ $7.7 \mathrm{~Hz}, 2 \mathrm{H}$ ), 2.25 (quintet, $J=7.7 \mathrm{~Hz}, 2 \mathrm{H}), 2.04(\mathrm{~m}, 2 \mathrm{H}), 1.41(\mathrm{~m}, 2 \mathrm{H}), 0.90$ (t, $J=7.4$ $\mathrm{Hz}, 3 \mathrm{H}) ;{ }^{13} \mathrm{C} \mathrm{NMR}\left(125 \mathrm{MHz}, \mathrm{CDCl}_{3}\right) \delta 186.7,136.4,124.1,71.3,52.4,50.6,34.3,22.0$, 14.7, 13.6; IR (thin film) 1507, 1472, $1443 \mathrm{~cm}^{-1}$; HRMS (ES+) m/z calcd for $\mathrm{C}_{10} \mathrm{H}_{17} \mathrm{NOSNa}(\mathrm{M}+\mathrm{Na})$ 222.0928, found 222.0929; LRMS (ES+) m/z fragments 200.0, 195.1, 150.0.

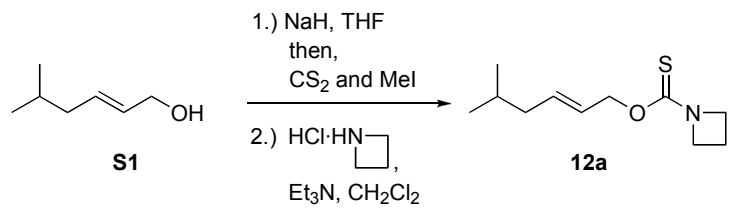

(E)-O-5-Methyl-2-hexenyl 1-azetinylcarbamothioate (12a). Following the procedure described for the preparation of 10c, alcohol S1 $(0.95 \mathrm{~g}, 8.3 \mathrm{mmol})$ was converted to the corresponding methylxanthate $(1.38 \mathrm{~g}, 81 \%$ yield). Azetidine hydrochloride ( $252 \mathrm{mg}, 2.69 \mathrm{mmol}, 2.5$ equiv) was added to a solution of a portion (500 $\mathrm{mg}, 1.06 \mathrm{mmol})$ of this crude methylxanthate and $\mathrm{CH}_{2} \mathrm{Cl}_{2}(10 \mathrm{~mL})$. Triethylamine $(375$ $\mathrm{mL}, 2.70 \mathrm{mmol}, 2.5$ equiv) was added and the resulting solution was maintained at room 
temperature for $30 \mathrm{~min}$. Concentration and purification of the residue by silica gel chromatography $\left(5 \% \mathrm{Et}_{2} \mathrm{O}\right.$ :hexanes) provided $O$-carbamothioate $12 \mathrm{a}(462 \mathrm{mg}, 88 \%)$ as a colorless oil: $R_{f} 0.28$ (5\% Et $2 \mathrm{O}$ :hexanes); ${ }^{1} \mathrm{H} \mathrm{NMR}\left(500 \mathrm{MHz}, \mathrm{CDCl}_{3}\right) \delta 5.76$ (dt, $J=$ $16.2,8.1 \mathrm{~Hz}, 1 \mathrm{H}), 5.61(\mathrm{dt}, J=16.2,6.4 \mathrm{~Hz}, 1 \mathrm{H}), 4.87(\mathrm{~d}, J=6.4 \mathrm{~Hz}, 2 \mathrm{H}), 4.19(\mathrm{t}, J=7.6$ $\mathrm{Hz}, 2 \mathrm{H}), 4.10(\mathrm{t}, J=7.6 \mathrm{~Hz}, 2 \mathrm{H}), 2.25(\mathrm{~m}, 2 \mathrm{H}), 1.95$ (app. t, $J=7.0 \mathrm{~Hz}, 2 \mathrm{H}), 1.64(\mathrm{~m}$, $1 \mathrm{H}), 0.89(\mathrm{~d}, J=6.7 \mathrm{~Hz}, 6 \mathrm{H}) ;{ }^{13} \mathrm{C} \mathrm{NMR}\left(125 \mathrm{MHz}, \mathrm{CDCl}_{3}\right) \delta 186.7,135.4,125.1,71.3$, 52.4, 50.6, 41.6, 28.1, 22.3, 14.8; IR (thin film) 1499, 1468, $1443 \mathrm{~cm}^{-1}$; HRMS (ES+) m/z calcd for $\mathrm{C}_{11} \mathrm{H}_{19} \mathrm{NOSNa}(\mathrm{M}+\mathrm{Na}) 236.1085$, found 236.1094; LRMS (ES+) m/z fragments 214.2, 150.1, 118.0.

(E)-O-4-(tert-Butyldimethylsilyloxy)but-2-enyl 1-azetinylcarbamothioate (12b). To a solution of (E)-4-(tert-butyldimethylsilyloxy)but-2-en-1-ol ${ }^{3}(0.37 \mathrm{~g}, 1.8$ $\mathrm{mmol})$ and $\mathrm{CS}_{2}(158 \mathrm{~mL})$ at $0{ }^{\circ} \mathrm{C}$ was added $\mathrm{NaH}(0.87 \mathrm{~g}$ of $60 \% \mathrm{NaH}$ in mineral oil, 22 mmol, 12 equiv) in one portion. The solution was allowed to warm to $\mathrm{rt}$, and after $4 \mathrm{~h}$ $\mathrm{CH}_{3} \mathrm{I}$ (5.3 mL, $85 \mathrm{mmol}, 47$ equiv) was added dropwise. After $30 \mathrm{~min}$, saturated aqueous $\mathrm{NH}_{4} \mathrm{Cl}(200 \mathrm{~mL})$ was added to the solution, and the mixture was extracted with $\mathrm{CH}_{2} \mathrm{Cl}_{2}$ $(3 \times 75 \mathrm{~mL})$. The combined organic extracts were washed with $\mathrm{H}_{2} \mathrm{O}(100 \mathrm{~mL})$, brine $(100$ $\mathrm{mL})$, and the combined organic extracts were dried $\left(\mathrm{Na}_{2} \mathrm{SO}_{4}\right)$ and concentrated under reduced pressure. Purification by silica chromatography (hexanes, 5:1 hexanes:Et ${ }_{2} \mathrm{O}$ ) provided the xanthate $(0.359 \mathrm{~g}, 68 \%$ yield $)$ as a yellow oil.

To a solution of the xanthate prepared from the first step $(359 \mathrm{mg}, 1.23 \mathrm{mmol})$ and $\mathrm{CH}_{2} \mathrm{Cl}_{2}(7.4 \mathrm{~mL})$ at $\mathrm{rt}$ was added azetidine hydrochloride $(0.127 \mathrm{~g}, 1.35 \mathrm{mmol}, 1.1$ equiv), followed by $\mathrm{Et}_{3} \mathrm{~N}(0.19 \mathrm{~mL}, 1.4 \mathrm{mmol}, 1.1$ equiv). After $2 \mathrm{~h}$, the mixture was concentrated under reduced pressure. Purification by silica chromatography $(5 \%$ $\mathrm{Et}_{2} \mathrm{O}$ :hexanes, $10 \% \mathrm{Et}_{2} \mathrm{O}$ :hexanes) provided $O$-carbamothioate 12b (383 $\mathrm{mg}$, quant.) as a colorless oil: $R_{f} 0.23$ (5\% Et ${ }_{2} \mathrm{O}$ :hexanes); ${ }^{1} \mathrm{H} \mathrm{NMR}\left(500 \mathrm{MHz}, \mathrm{CDCl}_{3}\right) \delta 5.87(\mathrm{~m}, 2 \mathrm{H})$, $4.93(\mathrm{dt}, J=5.9,1.2 \mathrm{~Hz}, 2 \mathrm{H}), 4.20(\mathrm{~d}, J=2.7 \mathrm{~Hz}, 2 \mathrm{H}), 4.20(\mathrm{t}, J=7.6 \mathrm{~Hz}, 2 \mathrm{H}), 4.11(\mathrm{t}, J$ $=7.6 \mathrm{~Hz}, 2 \mathrm{H}), 2.26$ (quintet, $J=7.6 \mathrm{~Hz}, 2 \mathrm{H}), 0.92(\mathrm{~s}, 9 \mathrm{H}), 0.08(\mathrm{~s}, 6 \mathrm{H}) ;{ }^{13} \mathrm{C} \mathrm{NMR}(125$ $\left.\mathrm{MHz}, \mathrm{CDCl}_{3}\right) \delta 186.5,134.0,123.8,70.4,63.0,52.4,50.6,25.9,18.4,14.8,-5.3$; IR (thin film) $1501,1472,1445 \mathrm{~cm}^{-1}$; HRMS (ES+) m/z calcd for $\mathrm{C}_{14} \mathrm{H}_{27} \mathrm{NO}_{2} \mathrm{SSiNa}(\mathrm{M}+\mathrm{Na})$ 324.1429, found 324.1422; LRMS (ES+) m/z fragments 269.2, 215.2, 170.1 .

(E)-O-4-(Triisopropylsilyloxy)but-2-enyl 1-azetinylcarbamothioate (12c). Following the procedure described for the preparation of $\mathbf{1 2 b}$, $(E)-4-$ (triisopropylsilyloxy)but-2-en-1-ol $(227 \mathrm{mg}, 0.932 \mathrm{mmol})$ was converted to the $O$ carbamothioate 12c (196 mg, 61\% yield), and was obtained as a colorless oil: $R_{f} 0.37$ (10:1 hexanes:EtOAc); ${ }^{1} \mathrm{H}$ NMR $\left(500 \mathrm{MHz}, \mathrm{CDCl}_{3}\right) \delta 5.89(\mathrm{~m}, 2 \mathrm{H}), 4.93(\mathrm{~m}, 2 \mathrm{H}), 4.28$ $(\mathrm{m}, 2 \mathrm{H}), 4.20(\mathrm{t}, J=7.7 \mathrm{~Hz}, 2 \mathrm{H}) 4.11(\mathrm{t}, J=7.7 \mathrm{~Hz}, 2 \mathrm{H}), 2.26$ (quintet, $J=7.7 \mathrm{~Hz}, 2 \mathrm{H}$ ), 1.15-1.07 (m, 21H); ${ }^{13} \mathrm{C}$ NMR $\left(125 \mathrm{MHz}, \mathrm{CDCl}_{3}\right) \delta 186.7,134.2,123.5,70.5,63.1,52.5$, 50.7, 18.1, 14.9, 12.1; IR (thin film) 1499, $1445 \mathrm{~cm}^{-1}$; HRMS (ES+) m/z calcd for $\mathrm{C}_{17} \mathrm{H}_{33} \mathrm{NO}_{2} \mathrm{SSiNa}(\mathrm{M}+\mathrm{Na})$ 366.1899, found 366.1901; LRMS (ES+) $\mathrm{m} / \mathrm{z}$ fragments 293.2, 249.2.

\footnotetext{
3 For the various procedures to prepare (E)-4-siloxy-2-butene-1-ol's, see: (a) Corlay, H.; Motherwell, W. B.; Pennell, A. M. K.; Shipman, M.; Slawin, A. M. Z.; William, D. J. Tetrahedron, 1996, 52, 4883-4902. (b) Braddock, D. C.; Bhuva, R.; Millan, D. S.; Pérez-Fuertes, Y.; Roberts, C. A.; Sheppard, R. N.; Solanki, S.; Stokes, E. S. E.; White, A. J. P. Org. Lett. 2007, 9, 445-448. (c) Vlieghe, P.; Clerc, T.; Pannecouque, C.; Witvrouw, M.; Clercq, E. D.; Salles, J.-P.; Kraus, J.-L. J. Med. Chem. 2001, 44, 3014-3021. (d) Organ, M. G.; Cooper, J. T.; Rogers, L. R.; Soleymanzadeh, F.; Paul, T. J. Org. Chem. 2000, 65, 7959-7970.
} 


\section{(E)-O-4-(tert-Butyldiphenylsilyloxy)but-2-enyl}

1-azetinylcarbamothioate (12d). Following the procedure described for the preparation of 12b, (E)-4-(tertbutyldiphenylsilyloxy)but-2-en-1-ol $(214 \mathrm{mg}, 0.656 \mathrm{mmol})$ was converted to the $O$ carbamothioate 12d (151 mg, 54\% yield), and was obtained as a colorless oil: $R_{f} 0.50$ (5:1 hexanes:EtOAc); ${ }^{1} \mathrm{H}$ NMR $\left(500 \mathrm{MHz}, \mathrm{CDCl}_{3}\right) \delta 7.68(\mathrm{~d}, J=7.1 \mathrm{~Hz}, 4 \mathrm{H}), 7.45-7.38$ $(\mathrm{m}, 6 \mathrm{H}), 5.93(\mathrm{ddd}, J=15.5,5.7,5.7 \mathrm{~Hz}, 1 \mathrm{H}), 5.86(\mathrm{ddd}, J=15.6,3.8,3.8 \mathrm{~Hz}, 1 \mathrm{H}), 4.93$ $(\mathrm{d}, J=5.7 \mathrm{~Hz}, 2 \mathrm{H}), 4.23-4.19(\mathrm{~m}, 4 \mathrm{H}), 4.11(\mathrm{t}, J=7.7 \mathrm{~Hz}, 2 \mathrm{H}), 2.27$ (quintet, $J=7.7 \mathrm{~Hz}$, 2H), $1.07(\mathrm{~s}, 9 \mathrm{H}) ;{ }^{13} \mathrm{C}$ NMR $\left(125 \mathrm{MHz}, \mathrm{CDCl}_{3}\right) \delta 186.8,135.7,133.7,133.7,129.9$, $127.9,124.0,70.6,63.8,52.6,50.8,27.0,19.5,15.0$; IR (thin film) 1507, 1472, $1443 \mathrm{~cm}^{-}$ ; HRMS (ES+) $\mathrm{m} / \mathrm{z}$ calcd for $\mathrm{C}_{24} \mathrm{H}_{31} \mathrm{NO}_{2} \mathrm{SSiNa}(\mathrm{M}+\mathrm{Na}) 448.1743$, found 448.1737; LRMS (ES+) $\mathrm{m} / \mathrm{z}$ fragments 426.2, 302.2.

(E)-O-4-Hydroxybut-2-enyl 1-azetinylcarbamothioate (12e). TBAF (0.35 mL, $0.35 \mathrm{mmol}, 1 \mathrm{M}$ solution in THF, 1.1 equiv) was added to a $\mathrm{rt}$ solution of $O$ carbamothioate $12 \mathbf{b}(97 \mathrm{mg}, 0.32 \mathrm{mmol})$ in THF $(1 \mathrm{~mL})$. The solution was maintained at rt for $50 \mathrm{~min}$, then an additional aliquot of TBAF $(0.18 \mathrm{~mL}, 0.18 \mathrm{mmol}, 1 \mathrm{M}$ solution in THF, 0.5 equiv) was added. After $25 \mathrm{~min}$, the solution was diluted with $\mathrm{H}_{2} \mathrm{O}(20 \mathrm{~mL})$ and extracted with $\mathrm{Et}_{2} \mathrm{O}(3 \times 10 \mathrm{~mL})$. The combined organic layers were dried $\left(\mathrm{Na}_{2} \mathrm{SO}_{4}\right)$, and concentrated under reduced pressure (CAUTION: Product is Volatile!!). Purification by silica chromatography $\left(100 \% \mathrm{Et}_{2} \mathrm{O}\right)$ provided $O$-carbamothioate 12e $(47 \mathrm{mg}, 79 \%$ yield $)$ as a light yellow oil: $R_{f} 0.45\left(100 \% \mathrm{Et}_{2} \mathrm{O}\right) ;{ }^{1} \mathrm{H}$ NMR $\left(500 \mathrm{MHz}, \mathrm{CDCl}_{3}\right) \delta 5.95(\mathrm{dt}, J=$ 15.6, $4.8 \mathrm{~Hz}, 1 \mathrm{H}), 5.90$ (dt, $J=15.6,5.5 \mathrm{~Hz}, 1 \mathrm{H}), 4.96(\mathrm{~d}, J=5.6 \mathrm{~Hz}, 2 \mathrm{H}), 4.20(\mathrm{~m}, 4 \mathrm{H})$, $4.12(\mathrm{t}, J=7.8 \mathrm{~Hz}, 2 \mathrm{H}), 2.27$ (quintet, $J=7.7 \mathrm{~Hz}, 2 \mathrm{H}), 1.56(\mathrm{~m}, 1 \mathrm{H}) ;{ }^{13} \mathrm{C} \mathrm{NMR}(125$ $\left.\mathrm{MHz}, \mathrm{CDCl}_{3}\right) \delta 186.4,133.0,125.3,70.0,62.7,52.4,50.7,14.8$; IR (thin film) 3421 (br), 1511, 1472, $1445 \mathrm{~cm}^{-1}$; HRMS (ES+) $\mathrm{m} / \mathrm{z}$ calcd for $\mathrm{C}_{8} \mathrm{H}_{13} \mathrm{NO}_{2} \mathrm{SNa}(\mathrm{M}+\mathrm{Na}) 210.0565$, found 210.0558; LRMS (ES+) m/z fragments 195.2, 186.3, 167.1.

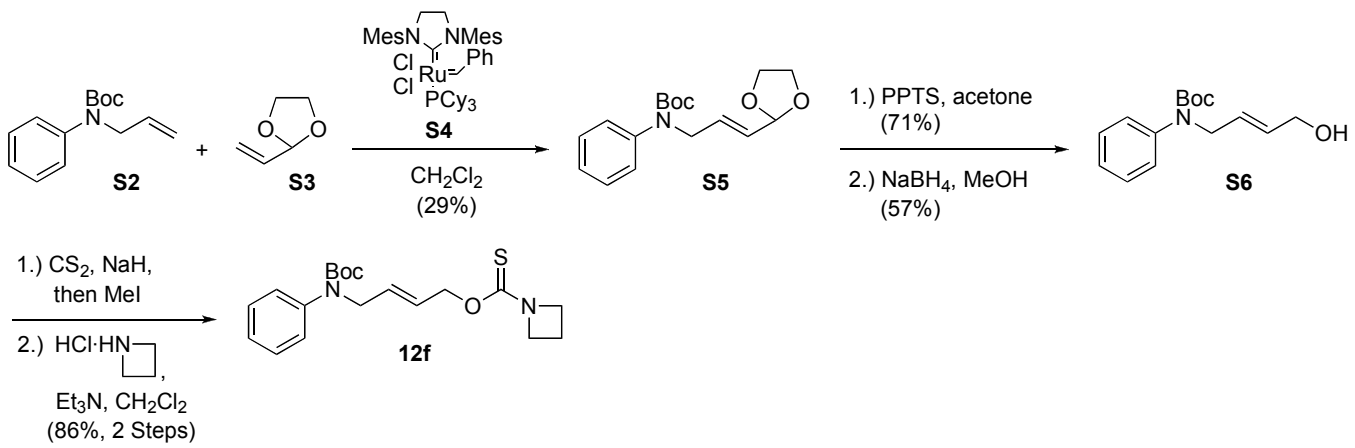

(E)-O-4-(tert-Butoxycarbonyl(phenyl)amino)but-2-enyl

1azetinylcarbamothioate (12f). A modification of a procedure reported by Grubbs and co-workers was employed. ${ }^{4,5}$ To a solution of terminal olefin $\mathbf{S 2}(1.0 \mathrm{~g}, 4.30 \mathrm{mmol})$ and $\mathrm{CH}_{2} \mathrm{Cl}_{2}(22 \mathrm{~mL})$ at $\mathrm{rt}$, was added vinyl dioxolane $\mathbf{S 3}(0.86 \mathrm{~mL}, 8.6 \mathrm{mmol}, 2$ equiv) and ruthenium catalyst $\mathbf{S} 4(0.092 \mathrm{~g}, 0.11 \mathrm{mmol}, 2.5 \mathrm{~mol} \%)$ in succession. The solution was heated to reflux for $18 \mathrm{~h}$. The solution was then allowed to cool to rt, and Celite was added. The mixture was concentrated under reduced pressure, and the Celite was loaded directly onto a silica column. Purification by silica chromatography (20:1

${ }^{4}$ O’Leary, D. J.; Blackwell, H. E., Washenfelder, R. A.; Miura, K.; Grubbs, R. H. Tetrahedron Lett. 1999, 40, 1091-1094.

${ }^{5}$ For the use of an allyl aniline substrate, see: Mennen, S. M. Ph. D. Dissertation, Boston College, 2007. 
hexanes:EtOAc, 10:1 hexanes:EtOAc, 5:1 hexanes:EtOAc) provided acetal S5 (380 mg, $29 \%$ yield) as a light yellow oil. Diagnostic data: ${ }^{1} \mathrm{H}$ NMR $\left(500 \mathrm{MHz}, \mathrm{CDCl}_{3}\right) \delta 7.32$ $(\operatorname{app~t}, J=7.8 \mathrm{~Hz}, 2 \mathrm{H}), 7.22-7.16(\mathrm{~m}, 3 \mathrm{H}), 6.02(\mathrm{ddd}, J=15.5,6.06 .0 \mathrm{~Hz}, 1 \mathrm{H}), 5.60(\mathrm{dd}$, $J=15.5,6.0 \mathrm{~Hz}, 1 \mathrm{H}), 5.28(\mathrm{~d}, J=6.0 \mathrm{~Hz}, 1 \mathrm{H}), 4.26(\mathrm{~d}, J=5.5 \mathrm{~Hz}, 2 \mathrm{H}), 3.99-3.87$ (m, $4 \mathrm{H}), 1.45(\mathrm{~s}, 9 \mathrm{H})$.

To a stirred solution of acetal $\mathbf{S 5}(0.38 \mathrm{~g}, 1.3 \mathrm{mmol})$ and acetone $(46 \mathrm{~mL})$ at $\mathrm{rt}$ was added pyridinium $p$-toluenesulfonate $(31 \mathrm{mg}, 0.13 \mathrm{mmol}, 0.1$ equiv) in one portion. The solution was heated to reflux for $17 \mathrm{~h}$, then allowed to cool to $\mathrm{rt}$ and concentrated under reduced pressure. Purification by silica chromatography (10:1 hexanes:EtOAc) provided the aldehyde (233 mg, 71\% yield) as a light yellow oil. Diagnostic data: ${ }^{1} \mathrm{H}$ NMR (500 $\left.\mathrm{MHz}, \mathrm{CDCl}_{3}\right) \delta 9.59(\mathrm{~d}, J=8.0 \mathrm{~Hz}, 1 \mathrm{H}), 7.34(\mathrm{app} \mathrm{t}, J=8.0 \mathrm{~Hz}, 2 \mathrm{H}), 7.23-7.20(\mathrm{~m}, 3 \mathrm{H})$, $6.88(\mathrm{ddd}, J=15.5,4.5,4.5 \mathrm{~Hz}, 1 \mathrm{H}), 6.24(\mathrm{dd}, J=15.5,7.5 \mathrm{~Hz}, 1 \mathrm{H}), 4.52(\mathrm{dd}, J=5.0$, $2.0 \mathrm{~Hz}, 2 \mathrm{H}), 1.45$ (s, 9H).

A solution of the aldehyde prepared in the previous step $(0.055 \mathrm{~g}, 0.21 \mathrm{mmol})$ and $\mathrm{MeOH}(1 \mathrm{~mL})$ was cooled to $0{ }^{\circ} \mathrm{C}$. $\mathrm{NaBH}_{4}(0.016 \mathrm{~g}, 0.42 \mathrm{mmol}, 2$ equiv) was added in one portion, and the solution was maintained at $0{ }^{\circ} \mathrm{C}$ for $2 \mathrm{~h}$. Acetone $(2 \mathrm{~mL})$ and saturated aqueous $\mathrm{NH}_{4} \mathrm{Cl}(10 \mathrm{~mL})$ were added to the solution, and the mixture was extracted with 1:1 hexanes:EtOAc $(3 \times 10 \mathrm{~mL})$. The combined organic layers were dried $\left(\mathrm{Na}_{2} \mathrm{SO}_{4}\right)$, and concentrated under reduced pressure. Purification by silica chromatography (1:1 hexanes:EtOAc) provided alcohol S6 (32 $\mathrm{mg}, 57 \%$ yield) as a light yellow oil. Diagnostic data: ${ }^{1} \mathrm{H}$ NMR $\left(600 \mathrm{MHz} \mathrm{CDCl}_{3}\right) \delta 7.31($ app t, $J=7.8 \mathrm{~Hz}, 2 \mathrm{H})$, 7.21-7.17 (m, 3H), 5.80 (ddd, $J=15.6,6.0,6.0 \mathrm{~Hz}, 1 \mathrm{H}), 5.74$ (ddd, $J=15.6,4.8,4.8 \mathrm{~Hz}$, $1 \mathrm{H}), 4.23(\mathrm{~d}, J=5.4 \mathrm{~Hz}, 2 \mathrm{H}), 4.12(\mathrm{~d}, J=4.8 \mathrm{~Hz}, 2 \mathrm{H}), 1.44(\mathrm{~s}, 9 \mathrm{H})$.

To a solution of alcohol S6 $(32 \mathrm{mg}, 0.12 \mathrm{mmol})$ and $\mathrm{CS}_{2}(9.8 \mathrm{~mL})$ at $0{ }^{\circ} \mathrm{C}$ was added $\mathrm{NaH}$ (59 $\mathrm{mg}$ of $60 \% \mathrm{NaH}$ in mineral oil, $1.4 \mathrm{mmol}, 12$ equiv) in one portion. The solution was allowed to warm to $\mathrm{rt}$, and after $4 \mathrm{~h} \mathrm{CH}_{3} \mathrm{I}(0.36 \mathrm{~mL}, 5.6 \mathrm{mmol}, 47$ equiv) was added dropwise. After $30 \mathrm{~min}$, saturated aqueous $\mathrm{NH}_{4} \mathrm{Cl}(20 \mathrm{~mL})$ was added to the solution, and the mixture was extracted with $\mathrm{CH}_{2} \mathrm{Cl}_{2}(3 \times 10 \mathrm{~mL})$. The combined organic extracts were washed with $\mathrm{H}_{2} \mathrm{O}$, brine, dried $\left(\mathrm{Na}_{2} \mathrm{SO}_{4}\right)$, and concentrated under reduced pressure to provide the crude xanthate as a yellow oil, which was used without further purification.

To a solution of the crude xanthate prepared in the previous step $(0.12 \mathrm{mmol})$ and $\mathrm{CH}_{2} \mathrm{Cl}_{2}(0.5 \mathrm{~mL})$ at $\mathrm{rt}$, was added azetidine hydrochloride (12 $\mathrm{mg}, 0.13 \mathrm{mmol}, 1.1$ equiv), followed by $\mathrm{Et}_{3} \mathrm{~N}(0.019 \mathrm{~mL}, 0.13 \mathrm{mmol}, 1.1$ equiv). The mixture was maintained at $\mathrm{rt}$ for $2 \mathrm{~h}$, then concentrated under reduced pressure. Purification by silica chromatography (10:1 hexanes:EtOAc, 5:1 hexanes:EtOAc) provided $O$-carbamothioate $\mathbf{1 2 f}(37 \mathrm{mg}, 86 \%$ yield) as a light yellow oil: $R_{f} 0.42\left(3: 1\right.$ hexanes:EtOAc); ${ }^{1} \mathrm{H} \mathrm{NMR}\left(500 \mathrm{MHz}, \mathrm{CDCl}_{3}\right) \delta$ $7.31(\mathrm{t}, J=7.8 \mathrm{~Hz}, 2 \mathrm{H}), 7.21-7.17(\mathrm{~m}, 3 \mathrm{H}), 5.87$ (ddd, $J=15.5,5.8,5.8 \mathrm{~Hz}, 1 \mathrm{H}), 5.73$ $(\mathrm{ddd}, J=15.5,6.0,6.0 \mathrm{~Hz}, 1 \mathrm{H}), 4.90(\mathrm{~d}, J=6.0 \mathrm{~Hz}, 2 \mathrm{H}), 4.23(\mathrm{~d}, J=5.7 \mathrm{~Hz}, 2 \mathrm{H}), 4.18$ (t, $J=7.7 \mathrm{~Hz}, 2 \mathrm{H}), 4.07$ (t, $J=7.7 \mathrm{~Hz}, 2 \mathrm{H}), 2.26$ (quintet, $J=7.7 \mathrm{~Hz}, 2 \mathrm{H}), 1.44(\mathrm{~s}, 9 \mathrm{H})$; ${ }^{13} \mathrm{C}$ NMR $\left(125 \mathrm{MHz}, \mathrm{CDCl}_{3}\right) \delta 186.7(\mathrm{C}), 154.6(\mathrm{C}), 142.8(\mathrm{CH}), 130.9(\mathrm{CH}), 128.8$ $(\mathrm{CH}), 126.7(\mathrm{CH}), 126.1(\mathrm{CH}), 80.6(\mathrm{C}), 70.3\left(\mathrm{CH}_{2}\right), 52.6\left(\mathrm{CH}_{2}\right), 51.9\left(\mathrm{CH}_{2}\right), 50.8\left(\mathrm{CH}_{2}\right)$, $28.5\left(\mathrm{CH}_{3}\right), 15.0\left(\mathrm{CH}_{2}\right)$; IR (thin film) 1698, 1598, $1499 \mathrm{~cm}^{-1} ; \mathrm{HRMS}(\mathrm{ES}+) \mathrm{m} / \mathrm{z}$ calcd for $\mathrm{C}_{19} \mathrm{H}_{26} \mathrm{~N}_{2} \mathrm{O}_{3} \mathrm{SNa}(\mathrm{M}+\mathrm{Na}) 385.1562$, found 385.1550; LRMS (ES+) $\mathrm{m} / \mathrm{z}$ fragments $360.3,323.2,293.2$. 


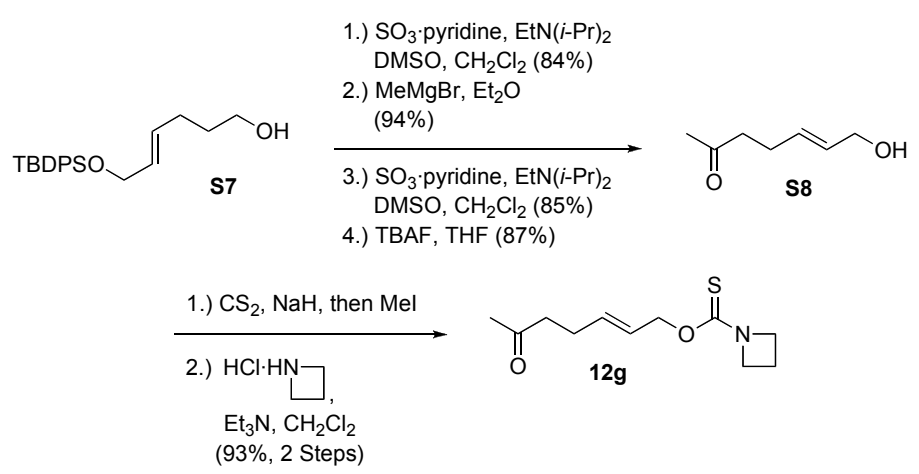

(E)-O-6-Oxohept-2-enyl 1-azetinylcarbamothioate (12g). A solution of alcohol $\mathbf{S 7}^{6}$ (279 $\left.\mathrm{mg}, 0.790 \mathrm{mmol}\right)$ and $\mathrm{CH}_{2} \mathrm{Cl}_{2}(8 \mathrm{~mL})$ was cooled to $0{ }^{\circ} \mathrm{C}$. $\mathrm{EtN}(i-\mathrm{Pr})_{2}(0.96 \mathrm{~mL}$, $5.5 \mathrm{mmol}, 7$ equiv) was added dropwise to the solution. After $10 \mathrm{~min}$, DMSO $(0.55 \mathrm{~mL}$, $7.9 \mathrm{mmol}, 10$ equiv) was added dropwise to the solution. After an additional $10 \mathrm{~min}$, $\mathrm{SO}_{3}$.pyridine ( $0.50 \mathrm{~g}, 3.2 \mathrm{mmol}, 4$ equiv) was added in one portion, and the solution was maintained at $0{ }^{\circ} \mathrm{C}$ for $45 \mathrm{~min}$. Saturated aqueous $\mathrm{NaHCO}_{3}(25 \mathrm{~mL})$ was added to the solution, and the mixture was allowed to warm to rt. The mixture was extracted with $\mathrm{CH}_{2} \mathrm{Cl}_{2}(3 \times 25 \mathrm{~mL})$, and the combined organic layers were dried $\left(\mathrm{Na}_{2} \mathrm{SO}_{4}\right)$, and concentrated under reduced pressure. Purification by silica chromatography (3:1 hexanes:EtOAc) provided the aldehyde (232 $\mathrm{mg}, 84 \%$ yield) as a colorless oil. Diagnostic data: ${ }^{1} \mathrm{H}$ NMR $\left(500 \mathrm{MHz}, \mathrm{CDCl}_{3}\right) \delta 9.78(\mathrm{~s}, 1 \mathrm{H}), 7.68-7.66(\mathrm{~m}, 4 \mathrm{H}), 7.45-7.37(\mathrm{~m}, 6 \mathrm{H})$, 5.70-5.58 (m, 2H), $4.16(\mathrm{dd}, J=6.0,1.5 \mathrm{~Hz}, 2 \mathrm{H}), 2.53-2.50(\mathrm{~m}, 2 \mathrm{H}), 2.40-2.37(\mathrm{~m}, 2 \mathrm{H})$, $1.06(\mathrm{~s}, 9 \mathrm{H})$.

A solution of the aldehyde prepared in the previous step $(214 \mathrm{mg}, 0.608 \mathrm{mmol})$ and $\mathrm{Et}_{2} \mathrm{O}(3 \mathrm{~mL})$ was cooled to $-78^{\circ} \mathrm{C}$. After the dropwise addition of $\mathrm{MeMgBr}(1.0 \mathrm{~mL}$, $3.0 \mathrm{mmol}, 3.0 \mathrm{M}$ in $\mathrm{Et}_{2} \mathrm{O}, 5$ equiv), the Dry Ice/acetone bath was allowed to slowly warm to rt. After $12 \mathrm{~h}, 0.5 \mathrm{M} \mathrm{HCl}(10 \mathrm{~mL})$ was slowly added to the solution, and the mixture was extracted with 3:1 hexanes:EtOAc $(3 \times 25 \mathrm{~mL})$. The combined organic layers were dried $\left(\mathrm{Na}_{2} \mathrm{SO}_{4}\right)$, and concentrated under reduced pressure. Purification by silica chromatography (5:1 hexanes:EtOAc) provided the secondary alcohol $(210 \mathrm{mg}, 94 \%$ yield) as a colorless oil. Diagnostic data: ${ }^{1} \mathrm{H} \mathrm{NMR}\left(500 \mathrm{MHz}, \mathrm{CDCl}_{3}\right) \delta 7.69(\operatorname{app~d}, J=$ $7.0 \mathrm{~Hz}, 4 \mathrm{H}), 7.45-7.37(\mathrm{~m}, 6 \mathrm{H}), 5.71-5.58(\mathrm{~m}, 2 \mathrm{H}), 4.17(\mathrm{~d}, J=4.5 \mathrm{~Hz}, 2 \mathrm{H}), 3.85-3.79$ $(\mathrm{m}, 1 \mathrm{H}), 2.19-2.06(\mathrm{~m}, 2 \mathrm{H}), 1.59-1.49(\mathrm{~m}, 2 \mathrm{H}), 1.21(\mathrm{~d}, J=6.5 \mathrm{~Hz}, 3 \mathrm{H}), 1.07(\mathrm{~s}, 9 \mathrm{H})$.

A solution of the secondary alcohol prepared in the previous step (397 mg, 1.08 mmol $)$ and $\mathrm{CH}_{2} \mathrm{Cl}_{2}(11 \mathrm{~mL})$ was cooled to $0{ }^{\circ} \mathrm{C}$. $\mathrm{EtN}(i \text {-Pr })_{2}(1.3 \mathrm{~mL}, 7.6 \mathrm{mmol}, 7$ equiv $)$ was added dropwise to the solution. After $10 \mathrm{~min}$, DMSO $(0.75 \mathrm{~mL}, 11 \mathrm{mmol}, 10$ equiv) was added dropwise to the solution. After and additional $10 \mathrm{~min}, \mathrm{SO}_{3} \cdot$ pyridine $(0.69 \mathrm{~g}$, $4.3 \mathrm{mmol}, 4$ equiv) was added in one portion, and the solution was maintained at $0{ }^{\circ} \mathrm{C}$ for 45 min. Saturated aqueous $\mathrm{NaHCO}_{3}$ was added to the solution, and the mixture was allowed to warm to rt. The mixture was extracted with $\mathrm{CH}_{2} \mathrm{Cl}_{2}(3 \times 25 \mathrm{~mL})$, and the combined organic layers were dried $\left(\mathrm{Na}_{2} \mathrm{SO}_{4}\right)$, and concentrated under reduced pressure. Purification by silica chromatography (10:1 hexanes:EtOAc) provided the ketone (335mg, 85\% yield) as a colorless oil. Diagnostic data: ${ }^{1} \mathrm{H}$ NMR $\left(600 \mathrm{MHz}, \mathrm{CDCl}_{3}\right) \delta$ 7.68-7.67 (m, 4H), 7.44-7.37 (m, 6H), 5.66-5.56 (m, 2H), 4.16-4.15 (m, 2H), 2.49 (app t, $J=6.5 \mathrm{~Hz}, 2 \mathrm{H}), 2.32-2.29(\mathrm{~m}, 2 \mathrm{H}), 2.15(\mathrm{~s}, 3 \mathrm{H}), 1.06(\mathrm{~s}, 9 \mathrm{H})$.

\footnotetext{
${ }^{6}$ Hayashi, N.; Fujiwara, K.; Murai, A. Tetrahedron, 1997, 53, 12425-12468.
} 
To a solution of the ketone prepared in the previous step (168 $\mathrm{mg}, 0.459 \mathrm{mmol})$ and THF $(3 \mathrm{~mL})$ at $\mathrm{rt}$, was added TBAF $(0.69 \mathrm{~mL}, 0.69 \mathrm{mmol}, 1 \mathrm{M}$ in THF, 1.5 equiv). After $45 \mathrm{~min}$, dry silica powder was added to the solution and the slurry was concentrated under reduced pressure. The silica powder was loaded onto a silica column and purified by silica chromatography (5:1 hexanes:EtOAc, 1:1 hexanes:EtOAc) to provide alcohol S8 (52 mg, 87\% yield) as a colorless oil. Diagnostic data: ${ }^{1} \mathrm{H}$ NMR $\left(600 \mathrm{MHz}, \mathrm{CDCl}_{3}\right) \delta$ $5.68(\mathrm{~m}, 2 \mathrm{H}), 4.09(\mathrm{~d}, J=3.5 \mathrm{~Hz}, 2 \mathrm{H}), 2.54($ app t, $J=6.0 \mathrm{~Hz}, 2 \mathrm{H}), 2.34-2.31(\mathrm{~m}, 2 \mathrm{H})$, $2.15(\mathrm{~s}, 3 \mathrm{H})$.

Following the previously described procedure for $O$-carbamothioate 12f, alcohol S8 was converted to the $O$-carbamothioate 12g (42 $\mathrm{mg}, 93 \%$ yield), and was obtained as a light yellow oil: $R_{f} 0.28$ (3:1 hexanes:EtOAc); ${ }^{1} \mathrm{H} \mathrm{NMR}\left(500 \mathrm{MHz}, \mathrm{CD}_{2} \mathrm{Cl}_{2}\right) \delta 5.77$ (ddd, $J=15.4,6.6,6.6 \mathrm{~Hz}, 1 \mathrm{H}), 5.64(\mathrm{ddd}, J=15.4,6.2,6.2 \mathrm{~Hz}, 1 \mathrm{H}), 4.82(\mathrm{~d}, J=6.1$ $\mathrm{Hz}, 2 \mathrm{H}), 4.12$ (t, $J=7.7 \mathrm{~Hz}, 2 \mathrm{H}), 4.07(\mathrm{t}, J=7.7 \mathrm{~Hz}, 2 \mathrm{H}), 2.52(\mathrm{t}, J=7.4 \mathrm{~Hz}, 2 \mathrm{H}), 2.30$ $(\mathrm{q}, J=7.0 \mathrm{~Hz}, 2 \mathrm{H}), 2.23$ (quintet, $J=7.7 \mathrm{~Hz}, 2 \mathrm{H}), 2.11(\mathrm{~s}, 3 \mathrm{H}) ;{ }^{13} \mathrm{C} \mathrm{NMR}(125 \mathrm{MHz}$, $\left.\mathrm{CD}_{2} \mathrm{Cl}_{2}\right) \delta 208.0(\mathrm{C}), 187.1(\mathrm{C}), 134.7(\mathrm{CH}), 125.6(\mathrm{CH}), 71.2\left(\mathrm{CH}_{2}\right), 53.0\left(\mathrm{CH}_{2}\right), 51.2$ $\left(\mathrm{CH}_{2}\right), 43.0\left(\mathrm{CH}_{2}\right), 30.3\left(\mathrm{CH}_{3}\right), 26.8\left(\mathrm{CH}_{2}\right), 15.3\left(\mathrm{CH}_{2}\right)$; IR (thin film) 1742, 1717, 1698, $\mathrm{cm}^{-1}$; HRMS (ES+) $\mathrm{m} / \mathrm{z}$ calcd for $\mathrm{C}_{11} \mathrm{H}_{17} \mathrm{NO}_{2} \mathrm{SNa}(\mathrm{M}+\mathrm{Na}) 250.0878$, found 250.0887; LRMS (ES+) m/z fragments 215.1, 213.1, 173.1.

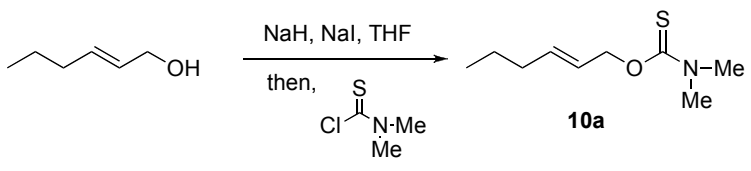

(E)-O-2-Hexenyl 1-dimethylcarbamothioate (10a). A modified procedure reported by Falck was employed. ${ }^{7}$ To a solution of trans-2-hexene-1-ol (1.2 mL, 1.0 $\mathrm{mmol})$ and THF $(20 \mathrm{~mL})$, was added to a solution of $\mathrm{NaH}(1.2 \mathrm{~g}$ of $60 \% \mathrm{NaH}$ in mineral oil, $30 \mathrm{mmol}, 3$ equiv) and THF $(40 \mathrm{~mL})$ at $0{ }^{\circ} \mathrm{C}$. The solution was maintained at $0{ }^{\circ} \mathrm{C}$ for $30 \mathrm{~min}$, then $\mathrm{NaI}(150 \mathrm{mg}, 0.998 \mathrm{mmol}, 0.1$ equiv) and $N, N$-dimethylthiocarbamoyl chloride (1.48 g, $12.0 \mathrm{mmol}, 1.2$ equiv) were added successively. The solution was allowed to warm to $\mathrm{rt}$ and was maintained at $\mathrm{rt}$ for $1 \mathrm{~h}$. Saturated aqueous $\mathrm{NH}_{4} \mathrm{Cl}$ was added to the solution, and the mixture was extracted with $\mathrm{Et}_{2} \mathrm{O}(3 \times 20 \mathrm{~mL})$. The combined organic layers were washed with $\mathrm{H}_{2} \mathrm{O}$, brine, dried $\left(\mathrm{MgSO}_{4}\right)$, and concentrated under reduced pressure. Purification by silica chromatography (5\% $\mathrm{Et}_{2} \mathrm{O}$ :hexanes) provided $O$-carbamothioate $\mathbf{1 0 a}(1.64 \mathrm{~g}, 88 \%$ yield $)$ as a pale yellow oil: $R_{f} 0.54(10: 1$ hexanes:EtOAc); ${ }^{1} \mathrm{H}$ NMR $\left(500 \mathrm{MHz}, \mathrm{CDCl}_{3}\right) \delta 5.80$ (ddd, $\left.J=15.4,6.7,6.7 \mathrm{~Hz}, 1 \mathrm{H}\right)$, 5.65 (ddd, $J=15.3,6.4,6.4 \mathrm{~Hz}, 1 \mathrm{H}), 4.91(\mathrm{~d}, J=6.3 \mathrm{~Hz}, 2 \mathrm{H}), 3.37(\mathrm{~s}, 3 \mathrm{H}), 3.12(\mathrm{~s}, 3 \mathrm{H})$, 2.05 (q, $J=7.1 \mathrm{~Hz}, 2 \mathrm{H}), 1.48$ (qn, $J=7.4 \mathrm{~Hz}, 2 \mathrm{H}), 0.90(\mathrm{t}, J=7.4 \mathrm{~Hz}, 3 \mathrm{H}) ;{ }^{13} \mathrm{C} \mathrm{NMR}$ $\left(125 \mathrm{MHz}, \mathrm{CDCl}_{3}\right) \delta 188.3(\mathrm{C}), 136.4(\mathrm{CH}), 124.4(\mathrm{CH}), 72.5\left(\mathrm{CH}_{2}\right), 42.9\left(\mathrm{CH}_{3}\right), 38.0$ $\left(\mathrm{CH}_{3}\right), 34.6\left(\mathrm{CH}_{2}\right), 22.3\left(\mathrm{CH}_{2}\right), 13.8\left(\mathrm{CH}_{3}\right)$; IR (thin film) 1522, $1393 \mathrm{~cm}^{-1}$; HRMS (ES+) $\mathrm{m} / \mathrm{z}$ calcd for $\mathrm{C}_{9} \mathrm{H}_{17} \mathrm{NOSNa}(\mathrm{M}+\mathrm{Na})$ 210.0928, found 210.0933; LRMS (ES+) $\mathrm{m} / \mathrm{z}$ fragments $188.1,173.1,167.1$.

(E)-O-4-(tert-Butyldimethylsilyloxy)but-2-enyl 1-dimethylcarbamothioate (13a). Following the procedure described for the preparation of 10a, 4-(tert-butyldimethyl-silanyloxy)-but-2-en-1-ol $(207 \mathrm{mg}, 1.03 \mathrm{mmol})$ was converted to the $O$ carbamothioate 13a (234 mg, 78\% yield), and was obtained as a pale yellow oil: $R_{f} 0.38$ (10:1 hexanes:EtOAc); ${ }^{1} \mathrm{H}$ NMR (500 MHz, $\left.\mathrm{CDCl}_{3}\right) \delta$ 5.89-5.88 (m, 2H), $4.97(\mathrm{~s}, 2 \mathrm{H})$,

${ }^{7}$ Barma, D. K.; Bandyopadhyay, A.; Capdevila, J. H.; Falk, J. R. Org. Lett. 2003, 5, 4755-4757. 
$4.21(\mathrm{~s}, 2 \mathrm{H}), 3.38(\mathrm{~s}, 3 \mathrm{H}), 3.13(\mathrm{~s}, 3 \mathrm{H}), 0.92(\mathrm{~s}, 9 \mathrm{H}), 0.08(\mathrm{~s}, 6 \mathrm{H}) ;{ }^{13} \mathrm{C} \mathrm{NMR}(125 \mathrm{MHz}$, $\left.\mathrm{CDCl}_{3}\right) \delta 188.2(\mathrm{C}), 134.0(\mathrm{CH}), 124.2(\mathrm{CH}), 71.6\left(\mathrm{CH}_{2}\right), 63.2\left(\mathrm{CH}_{2}\right), 43.0\left(\mathrm{CH}_{3}\right), 38.0$ $\left(\mathrm{CH}_{3}\right), 26.1\left(\mathrm{CH}_{3}\right), 18.6(\mathrm{C}),-5.0\left(\mathrm{CH}_{3}\right)$; IR (thin film) $1522,1463 \mathrm{~cm}^{-1}$; HRMS (ES+) $\mathrm{m} / \mathrm{z}$ calcd for $\mathrm{C}_{13} \mathrm{H}_{27} \mathrm{NO}_{2} \mathrm{SSiNa}(\mathrm{M}+\mathrm{Na}) 312.1429$, found 312.1429 ; LRMS $(\mathrm{ES}+) \mathrm{m} / \mathrm{z}$ fragment 290.2.

(E)-O-4-(tert-Butoxycarbonyl(phenyl)amino)but-2-enyl dimethylcarbamothioate (13b). To a solution of $\mathbf{S 6}(36 \mathrm{mg}, 0.14 \mathrm{mmol})$ and THF (0.6 $\mathrm{mL})$, was added $\mathrm{NaH}(10 \mathrm{mg}$ of $60 \% \mathrm{NaH}$ in mineral oil, $0.30 \mathrm{mmol}, 2.2$ equiv) neat at 0 ${ }^{\circ} \mathrm{C}$. The solution was maintained at $0{ }^{\circ} \mathrm{C}$ for $30 \mathrm{~min}$, then $\mathrm{NaI}(4 \mathrm{mg}, 0.03 \mathrm{mmol}, 0.2$ equiv) and $N, N$-dimethylthiocarbamoyl chloride (41 $\mathrm{mg}, 0.33 \mathrm{mmol}, 2.4$ equiv) were added successively. The solution was allowed to warm to $\mathrm{rt}$ and was maintained at $\mathrm{rt}$ for $1 \mathrm{~h}$. Saturated aqueous $\mathrm{NH}_{4} \mathrm{Cl}$ was added to the solution, and the mixture was extracted with $\mathrm{Et}_{2} \mathrm{O}(3 \times 10 \mathrm{~mL})$. The combined organic layers were washed with $\mathrm{H}_{2} \mathrm{O}$, brine, dried $\left(\mathrm{MgSO}_{4}\right)$, and concentrated under reduced pressure. Purification by silica chromatography (10:1 hexanes:EtOAc, 5:1 hexanes:EtOAc) provided $O$-carbamothioate 13b (41 mg, 85\% yield), and was obtained as a pale yellow oil. $R_{f} 0.38$ (3:1 hexanes:EtOAc); ${ }^{1} \mathrm{H}$ NMR (500 MHz, $\left.\mathrm{CDCl}_{3}\right) \delta 7.31(\mathrm{t}, J=7.8 \mathrm{~Hz}, 2 \mathrm{H}), 7.21-7.18(\mathrm{~m}$, $3 \mathrm{H}), 5.88(\mathrm{ddd}, J=15.5,5.7,5.7 \mathrm{~Hz}, 1 \mathrm{H}), 5.76(\mathrm{ddd}, J=15.4,5.9,5.9 \mathrm{~Hz}, 1 \mathrm{H}), 4.94(\mathrm{~d}, J$ $=5.9 \mathrm{~Hz}, 2 \mathrm{H}), 4.24(\mathrm{~d}, J=5.7 \mathrm{~Hz}, 2 \mathrm{H}), 3.36(\mathrm{~s}, 3 \mathrm{H}), 3.10(\mathrm{~s}, 3 \mathrm{H}), 1.44(\mathrm{~s}, 9 \mathrm{H}) ;{ }^{13} \mathrm{C} \mathrm{NMR}$ $\left(125 \mathrm{MHz}, \mathrm{CDCl}_{3}\right) \delta 188.0(\mathrm{C}), 154.6(\mathrm{C}), 142.8(\mathrm{C}), 130.8(\mathrm{CH}), 128.8(\mathrm{CH}), 126.7$ $(\mathrm{CH}), 126.7(\mathrm{CH}), 126.1(\mathrm{CH}), 80.6(\mathrm{C}), 71.3\left(\mathrm{CH}_{2}\right), 51.9\left(\mathrm{CH}_{2}\right), 43.0\left(\mathrm{CH}_{3}\right), 38.0\left(\mathrm{CH}_{3}\right)$, $28.5\left(\mathrm{CH}_{3}\right)$; IR (thin film) 1698, 1598, $1522 \mathrm{~cm}^{-1}$; HRMS (ES+) m/z calcd for $\mathrm{C}_{18} \mathrm{H}_{26} \mathrm{~N}_{2} \mathrm{O}_{3} \mathrm{SNa}(\mathrm{M}+\mathrm{Na})$ 373.1562, found 373.1569; LRMS (ES+) $\mathrm{m} / \mathrm{z}$ fragments 357.2 , 351.2, 279.2.

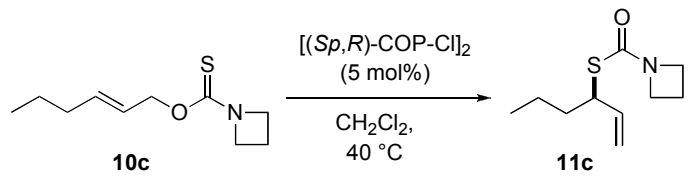

General procedure for rearrangement of $O$-allyl methyl- and 1azetidinylcarbamothioates using $5 \mathrm{~mol} \%[(\mathrm{Sp}, R)-\mathrm{COP}-\mathrm{Cl}]_{2}$ at $40{ }^{\circ} \mathrm{C}$. Preparation of $(R)-S$-1-Hexen-3-yl 1-azetinylcarbamothioate (11c). A solution of $S$-carbamothioate $10 c(53 \mathrm{mg}, 0.27 \mathrm{mmol}),[(S p, R)-\mathrm{COP}-\mathrm{Cl}]_{2}\left(18 \mathrm{mg}, 0.013 \mathrm{mmol}, 0.05\right.$ equiv) and $\mathrm{CH}_{2} \mathrm{Cl}_{2}$ $(0.51 \mathrm{~mL})$ was sealed, protected from light, and maintained at $40{ }^{\circ} \mathrm{C}$. After $13 \mathrm{~h}$, ethylenediamine $(0.010 \mathrm{~mL}, 0.13 \mathrm{mmol}, 0.5$ equiv) was added to the mixture after allowing it to cool to room temperature. After $15 \mathrm{~min}$, the orange solution was transferred to a round bottom flask with $\mathrm{CH}_{2} \mathrm{Cl}_{2}(10 \mathrm{~mL})$, and dry silica gel (ca. $60 \mathrm{mg}$ ) was added to the mixture. The slurry was concentrated, and the yellow powder was loaded onto a short silica gel column. Elution with 10:1 hexanes:EtOAc provided the transposed $S$-1-hexenyl 1-azetinylcarbamothioate (11c) (52 $\mathrm{mg}, 98 \%$ yield) as a light yellow oil. SFC analysis indicated an enantiomeric excess of $83 \%$ [OB column; flow $=1.5 \mathrm{~mL} / \mathrm{min} ; 10 \%$ isopropanol $/ 90 \% \mathrm{CO}_{2} ; \lambda=230 \mathrm{~nm}$; major enantiomer $\mathrm{t}_{\mathrm{R}}=4.24 \mathrm{~min}$; minor enantiomer $\left.\mathrm{t}_{\mathrm{R}}=3.88 \mathrm{~min}\right] ; R_{f} 0.22\left(10 \% \mathrm{Et}_{2} \mathrm{O}\right.$ :hexanes); ${ }^{1} \mathrm{H} \mathrm{NMR}\left(600 \mathrm{MHz}, \mathrm{CDCl}_{3}\right) \delta 5.79$ (ddd, $J=$ $18.2,10.1,8.3,1 \mathrm{H}), 5.22(\mathrm{~d}, J=17.0 \mathrm{~Hz}, 1 \mathrm{H}), 5.05(\mathrm{~d}, J=10.1 \mathrm{~Hz}, 1 \mathrm{H}), 4.03-4.00(\mathrm{~m}$, $5 \mathrm{H}), 2.29$ (quintet, $J=7.7 \mathrm{~Hz}, 2 \mathrm{H}), 1.70-1.61(\mathrm{~m}, 2 \mathrm{H}), 1.45-1.35(\mathrm{~m}, 2 \mathrm{H}), 0.91(\mathrm{t}, J=7.4$ $\mathrm{Hz}, 3 \mathrm{H}) ;{ }^{13} \mathrm{C}$ NMR $\left(125 \mathrm{MHz}, \mathrm{CDCl}_{3}\right) \delta 167.1(\mathrm{C}), 138.7(\mathrm{CH}), 115.4(\mathrm{CH}), 49.7\left(\mathrm{CH}_{2}\right)$, $46.7(\mathrm{CH}), 36.6\left(\mathrm{CH}_{2}\right), 20.2\left(\mathrm{CH}_{2}\right), 15.9\left(\mathrm{CH}_{2}\right), 13.8\left(\mathrm{CH}_{3}\right)$; IR (thin film) 1656, 1466, 
$1360 \mathrm{~cm}^{-1}$; HRMS (ES+) m/z calcd for $\mathrm{C}_{10} \mathrm{H}_{17} \mathrm{NOSNa}(\mathrm{M}+\mathrm{Na}) 222.0928$, found 222.0928; LRMS (ES+) $\mathrm{m} / \mathrm{z}$ fragments 200.0, 195.0; $[\alpha]^{24}{ }_{589}+25.0,[\alpha]^{24}{ }_{577}+26.0$, $[\alpha]_{546}^{24}+29.5,[\alpha]_{435}^{24}+51.1\left(c=2.41, \mathrm{CH}_{2} \mathrm{Cl}_{2}\right)$.

(S)-S-5-Methylhex-1-en-3-yl 1-azetinylcarbamothioate (14a). Following the general procedure described for the preparation of 11c and using $[(R p, S)-\mathrm{COP}-\mathrm{Cl}]_{2}, O$ carbamothioate 12a (54 mg, $0.25 \mathrm{mmol}$ ) was converted to the $S$-5-methylhex-1-en-3-yl 1azetinylcarbamothioate 14a (45 mg, 85\% yield), a light yellow oil: SFC analysis indicated an enantiomeric excess of $80 \%$ [OB column; flow: $1.0 \mathrm{~mL} / \mathrm{min} ; 10 \%$ isopropanol $/ 90 \% \mathrm{CO}_{2} ; \lambda=230 \mathrm{~nm}$; major enantiomer $\mathrm{t}_{\mathrm{R}}=4.75 \mathrm{~min}$; minor enantiomer $\left.\mathrm{t}_{\mathrm{R}}=5.30 \mathrm{~min}\right] ; R_{f} 0.33$ (20\% $\mathrm{Et}_{2} \mathrm{O}$ :pentane); ${ }^{1} \mathrm{H} \mathrm{NMR}\left(600 \mathrm{MHz}, \mathrm{CDCl}_{3}\right) \delta 5.77$ (ddd, $J=$ $17.1,8.5,8.5,1 \mathrm{H}), 5.24(\mathrm{~d}, J=17.0 \mathrm{~Hz}, 1 \mathrm{H}), 5.05(\mathrm{~d}, J=10.1 \mathrm{~Hz}, 1 \mathrm{H}), 4.09-4.04(\mathrm{~m}$, $5 \mathrm{H}), 2.29$ (quintet, $J=7.7 \mathrm{~Hz}, 2 \mathrm{H}), 1.74-1.67(\mathrm{~m}, 1 \mathrm{H}), 1.57-1.52(\mathrm{~m}, 2 \mathrm{H}), 0.92(\mathrm{~d}, J=4.5$ $\mathrm{Hz}, 3 \mathrm{H}), 0.91(\mathrm{~d}, J=4.3 \mathrm{~Hz}, 3 \mathrm{H}) ;{ }^{13} \mathrm{C} \mathrm{NMR}\left(125 \mathrm{MHz}, \mathrm{CDCl}_{3}\right) \delta 167.3(\mathrm{C}), 139.2(\mathrm{CH})$, $115.6(\mathrm{CH}), 50.0\left(\mathrm{CH}_{2}\right), 45.5(\mathrm{CH}), 43.6\left(\mathrm{CH}_{2}\right), 25.9(\mathrm{CH}), 22.9\left(\mathrm{CH}_{3}\right), 22.1\left(\mathrm{CH}_{3}\right), 16.2$ $\left(\mathrm{CH}_{2}\right)$; IR (thin film) 1656, 1468, $1364 \mathrm{~cm}^{-1}$; HRMS (ES+) m/z calcd for $\mathrm{C}_{11} \mathrm{H}_{19} \mathrm{NOSNa}$ $(\mathrm{M}+\mathrm{Na}) 236.1085$, found 236.1076; LRMS (ES+) $\mathrm{m} / \mathrm{z}$ fragments $214.2,173.1 ;[\alpha]^{24}{ }_{589}-$ 21.9, $[\alpha]^{25}{ }_{577}-22.6,[\alpha]^{25}{ }_{546}-25.6,[\alpha]^{24}{ }_{435}-44.3,[\alpha]^{25}{ }_{405}-51.5\left(c=0.55, \mathrm{CH}_{2} \mathrm{Cl}_{2}\right)$.

(R)-S-1-(tert-Butyldimethylsilyloxy)but-3-en-2-yl 1-azetinylcarbamothioate (14b). Following the general procedure described for the preparation of 11c and using $[(R p, S)-C O P-C l]_{2}, O$-carbamothioate $\mathbf{1 2 b}(95 \mathrm{mg}, 0.32 \mathrm{mmol})$ was converted to the $S$ - 1 (tert-butyldimethylsilyloxy)but-3-en-2-yl 1-azetinylcarbamothioate 14b (81 $\mathrm{mg}, 85 \%$ yield), and was obtained as a light yellow oil: SFC analysis indicated an enantiomeric excess of $88 \%$ [OB column; flow: $1.0 \mathrm{~mL} / \mathrm{min} ; 10 \%$ isopropanol $/ 90 \% \mathrm{CO}_{2} ; \lambda=230 \mathrm{~nm}$; major enantiomer $t_{R}=3.48 \mathrm{~min}$; minor enantiomer $\left.t_{R}=3.79 \mathrm{~min}\right] ; R_{f} 0.29(20 \%$ $\mathrm{Et}_{2} \mathrm{O}$ :hexanes); ${ }^{1} \mathrm{H}$ NMR $\left(600 \mathrm{MHz}, \mathrm{CDCl}_{3}\right) \delta 5.93$ (ddd, $\left.J=18.0,10.2,7.9 \mathrm{~Hz}, 1 \mathrm{H}\right)$, $5.32(\mathrm{~d}, J=17.1 \mathrm{~Hz}, 1 \mathrm{H}), 5.14(\mathrm{dd}, J=10.3,0.5 \mathrm{~Hz}, 1 \mathrm{H}), 4.15-4.12(\mathrm{~m}, 1 \mathrm{H}), 4.05-4.04$ $(\mathrm{m}, 4 \mathrm{H}), 3.84(\mathrm{dd}, J=10.1,4.6 \mathrm{~Hz}, 1 \mathrm{H}), 3.73(\mathrm{dd}, J=10.1,6.7 \mathrm{~Hz}, 1 \mathrm{H}), 2.30$ (quintet, $J$ $=7.7 \mathrm{~Hz}, 2 \mathrm{H}), 0.89(\mathrm{~s}, 9 \mathrm{H}), 0.06(\mathrm{~s}, 3 \mathrm{H}), 0.06(\mathrm{~s}, 3 \mathrm{H}) ;{ }^{13} \mathrm{C} \mathrm{NMR}\left(150 \mathrm{MHz}, \mathrm{CDCl}_{3}\right)$ $\delta 167.0(\mathrm{C}), 136.1(\mathrm{CH}), 117.3(\mathrm{CH}), 66.1\left(\mathrm{CH}_{2}\right), 50.0\left(\mathrm{CH}_{2}\right), 49.1(\mathrm{CH}), 26.0\left(\mathrm{CH}_{3}\right), 18.5$ (C), $16.2\left(\mathrm{CH}_{2}\right),-5.1\left(\mathrm{CH}_{3}\right),-5.1\left(\mathrm{CH}_{3}\right)$; IR (thin film) 1656, 1472, $1360 \mathrm{~cm}^{-1}$; HRMS (ES+) $\mathrm{m} / \mathrm{z}$ calcd for $\mathrm{C}_{14} \mathrm{H}_{27} \mathrm{NO}_{2} \mathrm{SSiNa}(\mathrm{M}+\mathrm{Na}) 324.1429$, found 324.1429; LRMS (ES+) $\mathrm{m} / \mathrm{z}$ fragments 302.2, 215.2; $[\alpha]^{24}{ }_{589}+31.3,[\alpha]^{25}{ }_{577}+34.0,[\alpha]_{546}^{25}+45.5(c=2.1$, $\mathrm{CH}_{2} \mathrm{Cl}_{2}$ ).

(R)-S-1-(Triisopropylsilyloxy)but-3-en-2-yl 1-azetinylcarbamothioate (14c). Following the general procedure described for the preparation of 11c and using $[(R p, S)$ $\mathrm{COP}-\mathrm{Cl}]_{2}, O$-carbamothioate $12 \mathrm{c}(59 \mathrm{mg}, 0.173 \mathrm{mmol})$ was converted to the $S$ (triisopropylsilyloxy)but-3-en-2-yl 1-azetinylcarbamothioate 14c (59 mg, 99\% yield), and was obtained as a light yellow oil: $R_{f} 0.42$ (5:1 hexanes:EtOAc); ${ }^{1} \mathrm{H}$ NMR $(500 \mathrm{MHz}$, $\left.\mathrm{CDCl}_{3}\right) \delta 5.99(\mathrm{ddd}, J=18.1,10.2,7.9 \mathrm{~Hz}, 1 \mathrm{H}), 5.39(\mathrm{~d}, J=17.1 \mathrm{~Hz}, 1 \mathrm{H}), 5.20(\mathrm{~d}, J=$ $10.3 \mathrm{~Hz}, 1 \mathrm{H}), 4.24(\operatorname{app~q}, J=11.8,7.0 \mathrm{~Hz}, 1 \mathrm{H}), 4.06(\operatorname{app~t}, J=7.3 \mathrm{~Hz}, 4 \mathrm{H}), 4.00$ (dd, $J$ $=9.5,4.5 \mathrm{~Hz}, 1 \mathrm{H}), 3.89(\mathrm{dd}, J=9.8,6.5 \mathrm{~Hz}, 1 \mathrm{H}), 2.30$ (quintet, $J=7.7 \mathrm{~Hz}, 2 \mathrm{H}), 1.13-$ $1.06(\mathrm{~m}, 21 \mathrm{H}) ;{ }^{13} \mathrm{C}$ NMR $\left(125 \mathrm{MHz}, \mathrm{CDCl}_{3}\right) \delta 167.2(\mathrm{C}), 136.3(\mathrm{CH}), 117.1(\mathrm{CH}), 66.5$ $\left(\mathrm{CH}_{2}\right), 50.0\left(\mathrm{CH}_{2}\right), 49.4(\mathrm{CH}), 18.2\left(\mathrm{CH}_{3}\right), 16.2(\mathrm{CH}), 12.2\left(\mathrm{CH}_{2}\right)$; IR (thin film) 1659, $1465,1362 \mathrm{~cm}^{-1}$; HRMS (ES+) m/z calcd for $\mathrm{C}_{17} \mathrm{H}_{33} \mathrm{NO}_{2} \mathrm{SSiNa}(\mathrm{M}+\mathrm{Na}) 366.1899$, found 366.1900; LRMS (ES+) m/z fragments 344.2, 249.2; $[\alpha]_{589}^{25}+12.1,[\alpha]_{577}^{25}+13.1$, $[\alpha]^{25}{ }_{546}+14.6,[\alpha]^{25}{ }_{435}+24.1,[\alpha]^{25}{ }_{405}+29.3\left(c=1.0, \mathrm{CH}_{2} \mathrm{Cl}_{2}\right)$. 
For SFC analysis, the TIPS protecting group was removed. To a solution of $\mathbf{1 4 c}$ $(12 \mathrm{mg}, 0.034 \mathrm{mmol})$ and $\mathrm{THF}(0.5 \mathrm{~mL})$ at $0{ }^{\circ} \mathrm{C}$, was added pyridine $(0.01 \mathrm{~mL})$ and HF-pyridine $(0.005 \mathrm{~mL})$. The solution was allowed to warm to rt. After $16 \mathrm{~h}$, saturated aqueous $\mathrm{NaHCO}_{3}(15 \mathrm{~mL})$ was slowly added to the solution, and the mixture was extracted with $\mathrm{Et}_{2} \mathrm{O}(3 \mathrm{x} 10 \mathrm{~mL})$. The combined organic layers were washed with saturated aqueous $\mathrm{CuSO}_{4}(15 \mathrm{~mL})$, dried $\left(\mathrm{Na}_{2} \mathrm{SO}_{4}\right)$, and concentrated under reduced pressure (CAUTION: Product is Volatile!!). Purification by silica chromatography $(100 \%$ $\left.\mathrm{Et}_{2} \mathrm{O}\right)$ provided alcohol 14e (4 mg, 67\% yield) as a light yellow oil. SFC analysis indicated an enantiomeric excess of $87 \%$ [OB column; flow: $1.5 \mathrm{~mL} / \mathrm{min} ; 10 \%$ isopropanol $/ 90 \% \mathrm{CO}_{2} ; \lambda=230 \mathrm{~nm}$; major enantiomer $t_{\mathrm{R}}=5.84 \mathrm{~min}$; minor enantiomer $\left.\mathrm{t}_{\mathrm{R}}=7.26 \mathrm{~min}\right]$.

(R)-S-1-(tert-Butyldiphenylsilyloxy)but-3-en-2-yl 1-azetinylcarbamothioate (14d). Following the general procedure described for the preparation of 11c and using $[(R p, S)-\mathrm{COP}-\mathrm{Cl}]_{2}, O$-carbamothioate $12 \mathrm{~d}(33 \mathrm{mg}, 0.078 \mathrm{mmol})$ was converted to the $S$ - 1 (tert-butyldiphenylsilyloxy)but-3-en-2-yl 1-azetinylcarbamothioate 14d (29 $\mathrm{mg}, 86 \%$ yield), and was obtained as a light yellow oil: $R_{f} 0.32$ (5:1 hexanes:EtOAc); ${ }^{1} \mathrm{H}$ NMR $\left(600 \mathrm{MHz}, \mathrm{CDCl}_{3}\right) \delta 7.69($ app t, $J=5.5 \mathrm{~Hz}, 4 \mathrm{H}), 7.45-7.37(\mathrm{~m}, 6 \mathrm{H}), 6.00$ (ddd, $J=17.4$, $10.2,8.1,1 \mathrm{H}), 5.35(\mathrm{~d}, J=17.1 \mathrm{~Hz}, 1 \mathrm{H}), 5.17(\mathrm{~d}, J=10.3 \mathrm{~Hz}, 1 \mathrm{H}), 4.27$ (app q, $J=13.1$, $5.8 \mathrm{~Hz}, 1 \mathrm{H}), 4.05(\operatorname{app~t}, J=7.1 \mathrm{~Hz}, 4 \mathrm{H}), 3.91(\mathrm{dd}, J=10.2,4.7 \mathrm{~Hz}, 1 \mathrm{H}), 3.83(\mathrm{dd}, J=$ $10.1,6.1 \mathrm{~Hz}, 1 \mathrm{H}), 2.30$ (quintet, $J=7.6 \mathrm{~Hz}, 2 \mathrm{H}), 1.07(\mathrm{~s}, 9 \mathrm{H}) ;{ }^{13} \mathrm{C} \mathrm{NMR}(125 \mathrm{MHz}$, $\left.\mathrm{CDCl}_{3}\right) \delta 166.9(\mathrm{C}), 136.2(\mathrm{CH}), 135.9(\mathrm{CH}), 135.9(\mathrm{CH}), 133.6(\mathrm{CH}), 129.8(\mathrm{CH}), 127.8$ $(\mathrm{CH}), 127.8(\mathrm{CH}), 117.3(\mathrm{CH}), 66.6\left(\mathrm{CH}_{2}\right), 50.0\left(\mathrm{CH}_{2}\right), 49.2(\mathrm{CH}), 27.0\left(\mathrm{CH}_{3}\right), 19.6(\mathrm{C})$, $16.2\left(\mathrm{CH}_{2}\right)$; IR (thin film) 1656, 1428, $1362 \mathrm{~cm}^{-1}$; HRMS (ES+) m/z calcd for $\mathrm{C}_{24} \mathrm{H}_{31} \mathrm{NO}_{2} \mathrm{SSiNa}(\mathrm{M}+\mathrm{Na})$ 448.1743, found 448.1734; LRMS (ES+) $\mathrm{m} / \mathrm{z}$ fragments 426.2, 337.3; $[\alpha]^{25}{ }_{589}+4.2,[\alpha]^{25}{ }_{577}+5.6,[\alpha]^{25}{ }_{546}+6.0,[\alpha]^{25}{ }_{435}-5.53\left(c=0.6, \mathrm{CH}_{2} \mathrm{Cl}_{2}\right)$.

For SFC analysis, the TBDPS protecting group was removed. To a solution of 14d $(13 \mathrm{mg}, 0.030 \mathrm{mmol})$ and THF $(0.5 \mathrm{~mL})$ at $0{ }^{\circ} \mathrm{C}$, was added pyridine $(0.009 \mathrm{~mL})$ and HF.pyridine $(0.005 \mathrm{~mL})$. The solution was allowed to warm to rt. After $16 \mathrm{~h}$, saturated aqueous $\mathrm{NaHCO}_{3}(15 \mathrm{~mL})$ was slowly added to the solution, and the mixture was extracted with $\mathrm{Et}_{2} \mathrm{O}(3 \mathrm{x} 10 \mathrm{~mL})$. The combined organic layers were washed with saturated aqueous $\mathrm{CuSO}_{4}(15 \mathrm{~mL})$, dried $\left(\mathrm{Na}_{2} \mathrm{SO}_{4}\right)$, and concentrated under reduced pressure (CAUTION: Product is Volatile!!). Purification by silica chromatography (100\% $\mathrm{Et}_{2} \mathrm{O}$ ) provided alcohol 14e (4 mg, 71\% yield) as a light yellow oil. SFC analysis indicated an enantiomeric excess of $76 \%$ [OB column; flow: $1.5 \mathrm{~mL} / \mathrm{min} ; 10 \%$ isopropanol $/ 90 \% \mathrm{CO}_{2} ; \lambda=230 \mathrm{~nm}$; major enantiomer $t_{\mathrm{R}}=5.80 \mathrm{~min}$; minor enantiomer $\left.\mathrm{t}_{\mathrm{R}}=7.44 \mathrm{~min}\right]$.

$(R)-S$-1-Hydroxybut-3-en-2-yl 1-azetinylcarbamothioate (14e). Following the general procedure described for the preparation of 11c and using $[(R p, S)-\mathrm{COP}-\mathrm{Cl}]_{2}, O-$ carbamothioate $12 \mathrm{e}(76 \mathrm{mg}, 0.41 \mathrm{mmol})$ was converted to the $S$-1-hydroxybut-3-en-2-yl 1-azetinylcarbamothioate 14e (42 mg, 55\% yield, CAUTION: Product is Volatile!!!), and was obtained as a light yellow oil: SFC analysis indicated an enantiomeric excess of $61 \%$ [OB column; flow: $1.5 \mathrm{~mL} / \mathrm{min} ; 10 \%$ isopropanol $/ 90 \% \mathrm{CO}_{2} ; \lambda=230 \mathrm{~nm}$; major enantiomer $\mathrm{t}_{\mathrm{R}}=5.81 \mathrm{~min}$; minor enantiomer $\left.\mathrm{t}_{\mathrm{R}}=7.15 \mathrm{~min}\right] ; R_{f} 0.45\left(100 \% \mathrm{Et}_{2} \mathrm{O}\right) ;{ }^{1} \mathrm{H}$ NMR $\left(500 \mathrm{MHz}, \mathrm{CDCl}_{3}\right) \delta 5.91(\mathrm{ddd}, J=17.4,10.3,7.8 \mathrm{~Hz}, 1 \mathrm{H}), 5.36(\mathrm{~d}, J=17.2 \mathrm{~Hz}$, $1 \mathrm{H}), 5.24$ (d, $J=10.4 \mathrm{~Hz}, 1 \mathrm{H}), 4.20($ app q, 13.0, $6.8 \mathrm{~Hz}, 1 \mathrm{H}), 4.09$ (app t, $J=7.6 \mathrm{~Hz}$, $4 \mathrm{H}), 3.88(\mathrm{dd}, J=11.2,5.4 \mathrm{~Hz}, 1 \mathrm{H}), 3.77(\mathrm{dd}, J=11.2,7.0 \mathrm{~Hz}, 1 \mathrm{H}), 2.33$ (quintet, $J=$ 7.6 Hz, 2H); ${ }^{13} \mathrm{C} \mathrm{NMR}\left(150 \mathrm{MHz}, \mathrm{CDCl}_{3}\right) \delta 167.0,134.2,118.4,65.7,50.0,49.2,30.3$, 
15.9; IR (thin film) 3404 (broad), 1636, $1372 \mathrm{~cm}^{-1}$; HRMS (ES+) m/z calcd for $\mathrm{C}_{8} \mathrm{H}_{13} \mathrm{NO}_{2} \mathrm{SNa}(\mathrm{M}+\mathrm{Na}) 210.0565$, found 210.0562; LRMS (ES+) $\mathrm{m} / \mathrm{z}$ fragments 188.1, $167.1 ;[\alpha]^{23}{ }_{589}-0.16,[\alpha]^{24}{ }_{577}-0.30,[\alpha]^{24}{ }_{546}-0.030,[\alpha]^{24}{ }_{435}-3.5\left(c=2.2, \mathrm{CH}_{2} \mathrm{Cl}_{2}\right)$.

(R)-S-1-(tert-Butoxycarbonyl(phenyl)amino)but-3-en-2-yl azetinylcarbamothioate (14f). Following the general procedure described for the preparation of 11c and using $[(R p, S)-\mathrm{COP}-\mathrm{Cl}]_{2}, O$-carbamothioate 12f $(23 \mathrm{mg}, 0.065$ mmol) was converted to the $S$-1-(tert-butoxycarbonyl(phenyl)amino)but-3-en-2-yl 1azetinylcarbamothioate $\mathbf{1 4 f}(16 \mathrm{mg}, 68 \%$ yield), and was obtained as a light yellow oil: SFC analysis indicated an enantiomeric excess of 71\% [AD column; flow: $1.5 \mathrm{~mL} / \mathrm{min}$; $10 \%$ isopropanol $/ 90 \% \mathrm{CO}_{2} ; \lambda=230 \mathrm{~nm}$; major enantiomer $\mathrm{t}_{\mathrm{R}}=7.92 \mathrm{~min}$; minor enantiomer $\left.\mathrm{t}_{\mathrm{R}}=7.39 \mathrm{~min}\right] ; R_{f} 0.31$ (3:1 hexanes:EtOAc); ${ }^{1} \mathrm{H}$ NMR $\left(500 \mathrm{MHz}, \mathrm{CDCl}_{3}\right) \delta$ 7.34 (app t, $J=7.7 \mathrm{~Hz}, 2 \mathrm{H}), 7.22-7.19$ (m, 3H), 5.84 (ddd, $J=17.7,9.0,9.0 \mathrm{~Hz}, 1 \mathrm{H})$, $5.27(\mathrm{~d}, J=17.0 \mathrm{~Hz}, 1 \mathrm{H}), 5.15(\mathrm{~d}, J=10.2 \mathrm{~Hz}, 1 \mathrm{H}), 4.19-4.09(\mathrm{~m}, 1 \mathrm{H}), 4.09-3.93(\mathrm{~m}$, $5 \mathrm{H}), 3.89(\mathrm{dd}, J=14.0,6.9 \mathrm{~Hz}, 1 \mathrm{H}), 2.28$ (quintet, $J=7.6 \mathrm{~Hz}, 2 \mathrm{H}), 1.44$ (brs, 9H); ${ }^{13} \mathrm{C}$ NMR (125 MHz, $\left.\mathrm{CDCl}_{3}\right) \delta 166.4(\mathrm{C}), 154.9(\mathrm{C}), 142.0(\mathrm{C}), 136.2(\mathrm{C}), 128.9(\mathrm{CH}), 127.6$ $(\mathrm{CH}), 126.5(\mathrm{CH}), 118.0(\mathrm{CH}), 80.7(\mathrm{C}), 52.5\left(\mathrm{CH}_{2}\right), 50.0\left(\mathrm{CH}_{2}\right), 46.1(\mathrm{CH}), 28.5\left(\mathrm{CH}_{3}\right)$, $16.2\left(\mathrm{CH}_{2}\right)$; IR (thin film) 1698, 1659, 1495, $1364 \mathrm{~cm}^{-1}$; HRMS (ES+) m/z calcd for $\mathrm{C}_{19} \mathrm{H}_{26} \mathrm{~N}_{2} \mathrm{O}_{3} \mathrm{SNa}(\mathrm{M}+\mathrm{Na}) 385.1562$, found 385.1559; LRMS (ES+) $\mathrm{m} / \mathrm{z}$ fragments 363.2, $263.1 ;[\alpha]^{25}{ }_{589}-21.1,[\alpha]^{25}{ }_{577}-22.3,[\alpha]^{25}{ }_{546}-25.2,[\alpha]^{24}{ }_{435}-46.7\left(c=0.70, \mathrm{CH}_{2} \mathrm{Cl}_{2}\right)$.

(S)-S-6-Oxohept-1-en-3-yl 1-azetinylcarbamothioate (14g). Following the general procedure described for the preparation of 11c and using $[(R p, S)-\mathrm{COP}-\mathrm{Cl}]_{2}, O-$ carbamothioate $12 \mathrm{~g}$ (15 mg, $0.07 \mathrm{mmol})$ was converted to the $S$-6-oxohept-1-en-3-yl 1azetinylcarbamothioate $\mathbf{1 4 g}$ (13 $\mathrm{mg}, 85 \%$ yield), and was obtained as a light yellow oil: $\mathrm{SFC}$ analysis indicated an enantiomeric excess of 76\% [OB column; flow: $1.5 \mathrm{~mL} / \mathrm{min}$; $10 \%$ isopropanol/ $90 \% \mathrm{CO}_{2} ; \lambda=230 \mathrm{~nm}$; major enantiomer $\mathrm{t}_{\mathrm{R}}=7.83 \mathrm{~min}$; minor enantiomer $\left.\mathrm{t}_{\mathrm{R}}=9.65 \mathrm{~min}\right] ; R_{f} 0.46\left(1: 1\right.$ hexanes:EtOAc); ${ }^{1} \mathrm{H} \mathrm{NMR}\left(600 \mathrm{MHz}, \mathrm{CD}_{2} \mathrm{Cl}_{2}\right) \delta$ 5.80 (ddd, $J=17.3,10.2,7.9 \mathrm{~Hz}, 1 \mathrm{H}), 5.20(\mathrm{~d}, J=17.1 \mathrm{~Hz}, 1 \mathrm{H}), 5.07$ (d, $J=10.2 \mathrm{~Hz}$, $1 \mathrm{H}), 4.01(\operatorname{app~t}, J=7.6 \mathrm{~Hz}, 4 \mathrm{H}), 3.93(\operatorname{app~q}, J=15.0,7.5 \mathrm{~Hz}, 1 \mathrm{H}), 2.52(\operatorname{app~t}, J=7.5$ $\mathrm{Hz}, 2 \mathrm{H}), 2.29$ (app quintet, $J=7.7,2 \mathrm{H}), 2.10$ (s, 3H), $1.96(\mathrm{ddd}, J=14.5,14.5,7.2 \mathrm{~Hz}$, $1 \mathrm{H}), 1.90(\mathrm{ddd}, J=14.8,14.8,7.5 \mathrm{~Hz}, 1 \mathrm{H}) ;{ }^{13} \mathrm{C} \mathrm{NMR}\left(125 \mathrm{MHz}, \mathrm{CDCl}_{3}\right) \delta 208.1(\mathrm{C})$, $166.8(\mathrm{C}), 138.9(\mathrm{CH}), 116.1(\mathrm{CH}), 50.4\left(\mathrm{CH}_{2}\right), 46.7(\mathrm{CH}), 41.4\left(\mathrm{CH}_{2}\right), 30.3\left(\mathrm{CH}_{3}\right), 28.9$ $\left(\mathrm{CH}_{2}\right), 16.5\left(\mathrm{CH}_{2}\right)$; IR (thin film) 1713, 1648, $1362 \mathrm{~cm}^{-1}$; HRMS (ES+) $\mathrm{m} / \mathrm{z}$ calcd for $\mathrm{C}_{11} \mathrm{H}_{17} \mathrm{NO}_{2} \mathrm{SNa}(\mathrm{M}+\mathrm{Na}) 250.0878$, found 250.0870; LRMS (ES+) m/z fragments 228.1, $217.1 ;[\alpha]^{24}{ }_{589}-16.9,[\alpha]^{24}{ }_{577}-17.4,[\alpha]^{25}{ }_{546}-18.4\left(c=0.23, \mathrm{CH}_{2} \mathrm{Cl}_{2}\right)$.

(R)-S-Hex-1-en-3-yl 1-dimethylcarbamothioate (11a). Following the general procedure described for the preparation of 11c and using $[(\mathrm{Sp}, R)-\mathrm{COP}-\mathrm{Cl}]_{2}, O-$ carbamothioate $10 \mathrm{a}(56 \mathrm{mg}, 0.30 \mathrm{mmol})$ was converted to the $S$-hex-1-en-3-yl 1dimethylcarbamothioate 11a (41 mg, 72\% yield), and was obtained as a light yellow oil: Chiral GC analysis indicated an enantiomeric excess of $82 \%$ [Cyclosil-B column; flow: $1.5 \mathrm{~mL} / \mathrm{min} ; 120{ }^{\circ} \mathrm{C}$, ; major enantiomer $\mathrm{t}_{\mathrm{R}}=22.6 \mathrm{~min}$; minor enantiomer $\left.\mathrm{t}_{\mathrm{R}}=21.8 \mathrm{~min}\right]$; $R_{f} 0.40$ (10:1 hexanes:EtOAc); ${ }^{1} \mathrm{H}$ NMR $\left(600 \mathrm{MHz}, \mathrm{CDCl}_{3}\right) \delta 5.83$ (ddd, $J=18.3,10.2$, $8.3 \mathrm{~Hz}, 1 \mathrm{H}), 5.25(\mathrm{~d}, J=17.0 \mathrm{~Hz}, 1 \mathrm{H}), 5.08(\mathrm{~d}, J=10.2 \mathrm{~Hz}, 1 \mathrm{H}), 4.05(\operatorname{app~q}, J=15.1$, $7.5 \mathrm{~Hz}, 1 \mathrm{H}), 3.00(\mathrm{~s}, 6 \mathrm{H}), 1.74-1.66(\mathrm{~m}, 2 \mathrm{H}), 1.47-1.40(\mathrm{~m}, 2 \mathrm{H}), 0.93(\mathrm{t}, J=7.4 \mathrm{~Hz}, 3 \mathrm{H})$; ${ }^{13} \mathrm{C}$ NMR $\left(125 \mathrm{MHz}, \mathrm{CDCl}_{3}\right) \delta 167.9(\mathrm{C}), 139.1(\mathrm{CH}), 115.7(\mathrm{CH}), 47.9(\mathrm{CH}), 36.8$ $\left(\mathrm{CH}_{3}\right), 36.8\left(\mathrm{CH}_{2}\right), 20.6\left(\mathrm{CH}_{2}\right), 14.0\left(\mathrm{CH}_{3}\right)$; IR (thin film) 1652, 1466, $1362 \mathrm{~cm}^{-1}$; HRMS (ES+) $\mathrm{m} / \mathrm{z}$ calcd for $\mathrm{C}_{9} \mathrm{H}_{17} \mathrm{NOSNa}(\mathrm{M}+\mathrm{Na}) 210.0928$, found 210.0925 ; LRMS (ES+) m/z 
fragments 188.1, 106.0; $[\alpha]^{24}{ }_{589}+39.1,[\alpha]^{24}{ }_{577}+40.3,[\alpha]^{24}{ }_{546}+45.6,[\alpha]^{24}{ }_{435}+83.2(c=$ $1.0, \mathrm{CH}_{2} \mathrm{Cl}_{2}$ ).

(R)-S-1-(tert-Butyldimethylsilyloxy)but-3-en-2-yl 1-dimethylcarbamothioate (15a). Following the general procedure described for the preparation of 11c and using $[(R p, S) \text {-COP-Cl }]_{2}, O$-carbamothioate $13 a(45 \mathrm{mg}, 0.22 \mathrm{mmol})$ was converted to the $S$ - 1 (tert-butyldimethylsilyloxy)but-3-en-2-yl 1-dimethylcarbamothioate 15a (44 mg, 97\% yield), and was obtained as a light yellow oil: $R_{f} 0.30$ (10:1 hexanes:EtOAc); ${ }^{1} \mathrm{H}$ NMR $\left(600 \mathrm{MHz}, \mathrm{CDCl}_{3}\right) \delta 5.95(\mathrm{ddd}, J=17.8,10.3,8.0 \mathrm{~Hz}, 1 \mathrm{H}), 5.34(\mathrm{~d}, J=17.1 \mathrm{~Hz}, 1 \mathrm{H})$, $5.16(\mathrm{~d}, J=10.3 \mathrm{~Hz}, 1 \mathrm{H}), 4.18(\operatorname{app~q}, J=12.2,6.7 \mathrm{~Hz}, 1 \mathrm{H}), 3.86(\mathrm{dd}, J=10.1,4.7 \mathrm{~Hz}$, $1 \mathrm{H}), 3.76(\mathrm{dd}, J=10.1,6.6 \mathrm{~Hz}, 1 \mathrm{H}), 3.00(\mathrm{~s}, 6 \mathrm{H}), 0.91(\mathrm{~s}, 9 \mathrm{H}), 0.07(\mathrm{~s}, 3 \mathrm{H}), 0.07(\mathrm{~s}, 3 \mathrm{H})$; ${ }^{13} \mathrm{C}$ NMR $\left(125 \mathrm{MHz}, \mathrm{CDCl}_{3}\right) \delta 167.4(\mathrm{C}), 136.0(\mathrm{CH}), 117.2(\mathrm{CH}), 65.8\left(\mathrm{CH}_{2}\right), 49.9$ $(\mathrm{CH}), 36.7\left(\mathrm{CH}_{3}\right), 25.9\left(\mathrm{CH}_{3}\right), 18.4(\mathrm{C}),-5.22\left(\mathrm{CH}_{3}\right),-5.23\left(\mathrm{CH}_{3}\right)$; IR (thin film) 1659, $1472,1362 \mathrm{~cm}^{-1}$; HRMS (ES+) m/z calcd for $\mathrm{C}_{13} \mathrm{H}_{27} \mathrm{NO}_{2} \mathrm{SSiNa}(\mathrm{M}+\mathrm{Na}) 312.1429$, found 312.1425; LRMS (ES+) m/z fragments 290.2, 269.1; $[\alpha]^{28}{ }_{589}+6.1,[\alpha]^{28}{ }_{577}+6.5,[\alpha]^{28}{ }_{546}$ $+7.3,[\alpha]^{28}{ }_{435}+6.1\left(c=0.98, \mathrm{CH}_{2} \mathrm{Cl}_{2}\right)$.

For GC analysis, the TBS protecting group was removed. To a solution of $\mathbf{1 5 a}(8$ $\mathrm{mg}, 0.04 \mathrm{mmol})$ and THF $(0.5 \mathrm{~mL})$ at $0{ }^{\circ} \mathrm{C}$, was added pyridine $(0.01 \mathrm{~mL})$ and HF-pyridine $(0.005 \mathrm{~mL})$. The solution was allowed to warm to rt. After $2 \mathrm{~h}$, saturated aqueous $\mathrm{NaHCO}_{3}(15 \mathrm{~mL})$ was slowly added to the solution, and the mixture was extracted with $\mathrm{Et}_{2} \mathrm{O}(3 \times 10 \mathrm{~mL})$. The combined organic layers were washed with saturated aqueous $\mathrm{CuSO}_{4}(15 \mathrm{~mL})$, dried $\left(\mathrm{Na}_{2} \mathrm{SO}_{4}\right)$, and concentrated under reduced pressure. Purification by silica chromatography $\left(100 \% \mathrm{Et}_{2} \mathrm{O}\right)$ provided the alcohol $(2 \mathrm{mg}$, $37 \%$ yield) as a light yellow oil. Chiral GC analysis indicated an enantiomeric excess of $88 \%$ [Cyclosil-B column; flow: $1.5 \mathrm{~mL} / \mathrm{min} ; 110^{\circ} \mathrm{C}$, ; major enantiomer $\mathrm{t}_{\mathrm{R}}=124.2 \mathrm{~min}$; minor enantiomer $\left.t_{R}=130.0 \mathrm{~min}\right]$

(R)-S-1-(tert-Butoxycarbonyl(phenyl)amino)but-3-en-2-yl dimethylcarbamothioate (15b). Following the general procedure described for the preparation of 11c and using $[(R p, S)-\mathrm{COP}-\mathrm{Cl}]_{2}, O$-carbamothioate 13b $(11 \mathrm{mg}, 0.031$ mmol) was converted to the $S$-1-(tert-butoxycarbonyl(phenyl)amino)but-3-en-2-yl 1dimethylcarbamothioate $\mathbf{1 5 b}(8 \mathrm{mg}, 77 \%$ yield $)$, and was obtained as a light yellow oil: SFC analysis indicated an enantiomeric excess of $81 \%$ [OB column; flow: $1.5 \mathrm{~mL} / \mathrm{min}$; $10 \%$ of $1: 1$ hexanes:isopropanol $/ 90 \% \mathrm{CO}_{2} ; \lambda=230 \mathrm{~nm}$; major enantiomer $\mathrm{t}_{\mathrm{R}}=8.83 \mathrm{~min}$; minor enantiomer $\left.\mathrm{t}_{\mathrm{R}}=11.41 \mathrm{~min}\right] ; R_{f} 0.35$ (3:1 hexanes:EtOAc); ${ }^{1} \mathrm{H}$ NMR $(600 \mathrm{MHz}$, $\left.\mathrm{CDCl}_{3}\right) \delta 7.34(\operatorname{app~t}, J=7.6 \mathrm{~Hz}, 2 \mathrm{H}), 7.22-7.19(\mathrm{~m}, 3 \mathrm{H}), 5.86(\mathrm{ddd}, J=17.6,9.4,9.4$ $\mathrm{Hz}, 1 \mathrm{H}), 5.29$ (d, $J=17.0 \mathrm{~Hz}, 1 \mathrm{H}), 5.16(\mathrm{~d}, J=10.2 \mathrm{~Hz}, 1 \mathrm{H}), 4.21-4.13(\mathrm{~m}, 1 \mathrm{H}), 4.09-$ $4.00(\mathrm{~m}, 1 \mathrm{H}), 3.91(\mathrm{dd}, J=14.1,7.0 \mathrm{~Hz}, 1 \mathrm{H}), 2.95(\mathrm{~s}, 6 \mathrm{H}), 1.26$ (brs, 9H); ${ }^{13} \mathrm{C}$ NMR $(125$ $\left.\mathrm{MHz} \mathrm{CDCl}_{3}\right) \delta 166.7(\mathrm{C}), 154.8(\mathrm{C}), 141.8(\mathrm{C}), 136.2(\mathrm{CH}), 128.8(\mathrm{CH}), 127.5(\mathrm{CH})$, $126.3(\mathrm{CH}), 117.8(\mathrm{CH}), 80.5(\mathrm{C}), 52.2\left(\mathrm{CH}_{2}\right), 46.9(\mathrm{CH}), 36.7\left(\mathrm{CH}_{3}\right), 28.3\left(\mathrm{CH}_{3}\right)$; IR (thin film) 1698, 1659, $1366 \mathrm{~cm}^{-1}$; HRMS (ES+) m/z calcd for $\mathrm{C}_{18} \mathrm{H}_{26} \mathrm{~N}_{2} \mathrm{O}_{3} \mathrm{SNa}(\mathrm{M}+\mathrm{Na})$ 373.1562, found 373.1556; LRMS (ES+) $\mathrm{m} / \mathrm{z}$ fragments 337.2, 251.1; $[\alpha]^{29}{ }_{589}-28.8$, $[\alpha]_{577}^{29}-29.6,[\alpha]_{546}^{29}-17.1,[\alpha]^{29}{ }_{435}-71.2\left(c=0.24, \mathrm{CH}_{2} \mathrm{Cl}_{2}\right)$. 


\section{The Absolute Configuration of $S$-1-Hexen-3-yl carbamothioates 11a-e.}
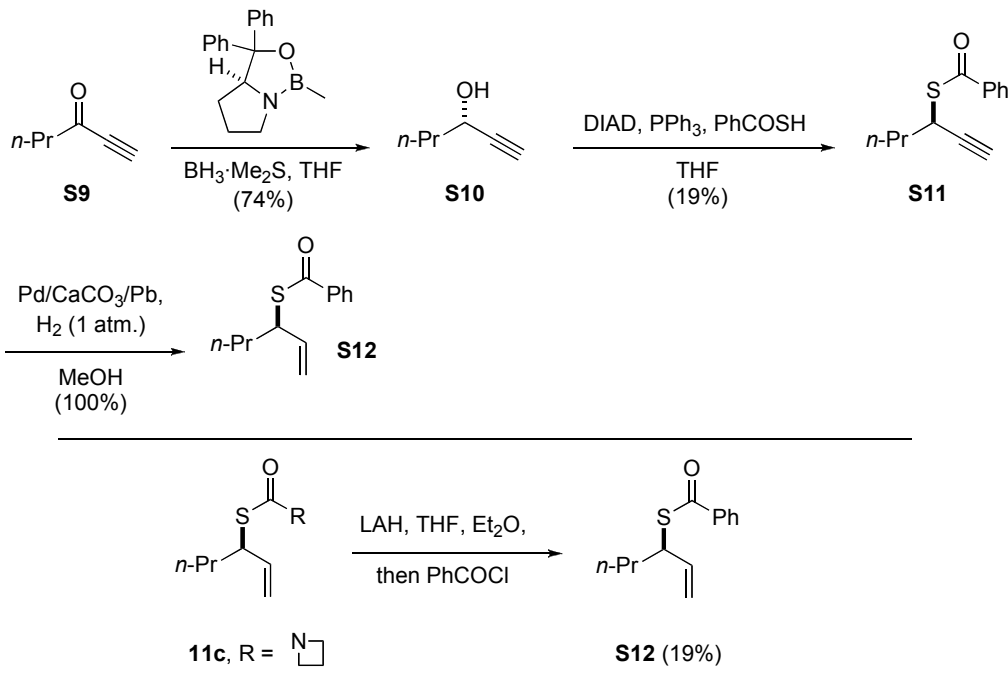

Conversion of Alcohol S10 to $(\boldsymbol{R})-\boldsymbol{S}$-Benzothioate $\mathbf{S 1 2}$. A solution of $(S)$-hex-1yn-3-ol $\mathbf{S 1 0} \mathbf{0}^{8}(22 \mathrm{mg}, 0.22 \mathrm{mmol})$ and THF $(1 \mathrm{~mL})$ was dried over activated $4 \AA$ molecular sieves for $10 \mathrm{~min}$. This solution was transferred to dry flask, and $S$-phenylthiotic acid (53 $\mu \mathrm{L}, 0.45$ mmol, 2 equiv), $\mathrm{Ph}_{3} \mathrm{P}$ (118 $\mathrm{mg}, 0.45 \mathrm{mmol}, 2$ equiv), and diisopropyl azodicarboxylate (64 $\mu \mathrm{L}, 0.45 \mathrm{mmol}, 2$ equiv) were added consecutively at room temperature. After $15 \mathrm{~h}$, the solution was diluted with $\mathrm{Et}_{2} \mathrm{O}(10 \mathrm{~mL})$, and the organic layer was washed with a saturated solution of $\mathrm{NaHCO}_{3}(10 \mathrm{~mL})$, brine $(10 \mathrm{~mL})$, dried $\left(\mathrm{MgSO}_{4}\right)$, and concentrated. Purification of the residue by silica gel chromatography $(1 \%$ $\mathrm{Et}_{2} \mathrm{O}$-hexanes, 2\% $\mathrm{Et}_{2} \mathrm{O}$-hexanes, 3\% $\mathrm{Et}_{2} \mathrm{O}$-hexanes, 5\% $\mathrm{Et}_{2} \mathrm{O}$-hexanes) provided alkyne S11 (9 mg, 19\% yield) as a colorless oil.

Lindlar catalyst $(1 \mathrm{mg}$ ) was added at room temperature to a solution of alkyne S11 (4 mg, $0.002 \mathrm{mmol})$ and $\mathrm{MeOH}(1 \mathrm{~mL})$. This mixture was placed under $\mathrm{H}_{2}$ (1 atm). After $1 \mathrm{~h}$, the mixture was filtered through Celite, the Celite was washed with $\mathrm{MeOH}(5 \mathrm{~mL})$, and the eluent was concentrated to provide $S$-benzothioate $\mathbf{S 1 2}$ (4 $\mathrm{mg}, 100 \%$ ) as a colorless oil. HPLC analysis indicated a enantiomeric excess of $66 \%$ [OB column; flow: $0.8 \mathrm{~mL} / \mathrm{min} ; 100 \%$ hexanes, major enantiomer $t_{R}=24.2 \mathrm{~min}$; minor enantiomer $t_{R}=18.6$ $\min ]$.

Conversion of $(R)-S$-1-Hexen-3-yl 1-azetinylcarbamothioate (11c) to $(R)-S$ Benzothioate S12. Solid $\mathrm{LiAlH}_{4}(29 \mathrm{mg}, 0.78 \mathrm{mmol})$ was added in one portion to a solution of $(R)-S$-1-hexen-3-yl 1-azetinylcarbamothioate (11c) $(77 \mathrm{mg}, 0.39 \mathrm{mmol})$, THF $(3 \mathrm{~mL})$ and $\mathrm{Et}_{2} \mathrm{O}(2 \mathrm{~mL})$ at room temperature. After $2 \mathrm{~h}$, the mixture was cooled to $0{ }^{\circ} \mathrm{C}$, and benzoyl chloride $(0.45 \mathrm{~mL}, 3.9 \mathrm{mmol})$ was added dropwise. The resulting mixture was allowed to warm to room temperature. After $30 \mathrm{~min}$, the mixture was filtered through Celite and the eluent was mixed with $\mathrm{Et}_{2} \mathrm{O}(10 \mathrm{~mL})$ and a saturated solution of $\mathrm{K}_{2} \mathrm{CO}_{3}(10$ $\mathrm{mL})$. The aqueous layer was separated, extracted with $\mathrm{Et}_{2} \mathrm{O}(3 \times 3 \mathrm{~mL})$, and the organic extract was dried $\left(\mathrm{MgSO}_{4}\right)$ and concentrated. Purification of the residue by silica gel chromatography (hexanes, 2\% $\mathrm{Et}_{2} \mathrm{O}$-hexanes, 5\% $\mathrm{Et}_{2} \mathrm{O}$-hexanes) provided $(R)-S$ -

${ }^{8}$ For the preparation of S11, see the Supporting Information of: Kirsch, S. F.; Overman, L. E.; White, N. S. Org. Lett. 2007, 9, 911-913. 
benzothiate S12 (16 mg, 19\%) as a colorless oil. HPLC analysis [OB column; flow: 0.8 $\mathrm{mL} / \mathrm{min} ; 100 \%$ hexanes, major enantiomer $t_{R}=24.1 \mathrm{~min}$; minor enantiomer $t_{R}=18.9$ $\min ]$.

In an identical fashion, $(R)-S-1$-hexen-3-yl carbamothioates 11a,b,d,e were correlated with $(R)$-S-benzothioate $\mathbf{S 1 2}$. 